\title{
Secrecy Generation for Multiaccess Channel Models
}

\author{
Imre Csiszár and Prakash Narayan
}

\begin{abstract}
Shannon theoretic secret key generation by several parties is considered for models in which a secure noisy channel with multiple input and output terminals and a public noiseless channel of unlimited capacity are available for accomplishing this goal. The secret key is generated for a set $A$ of terminals of the noisy channel, with the remaining terminals (if any) cooperating in this task through their public communication. Single-letter lower and upper bounds for secrecy capacities are obtained when secrecy is required from an eavesdropper that observes only the public communication and perhaps also a set of terminals disjoint from $A$. These bounds coincide in special cases, but not in general. We also consider models in which different sets of terminals share multiple keys, one for the terminals in each set with secrecy required from the eavesdropper as well as from the terminals not in this set. Partial results include showing links among the associated secrecy capacity region for multiple keys, the transmission capacity region of the multiple access channel defined by the secure noisy channel, and achievable rates for a single secret key for all the terminals.
\end{abstract}

Index Terms-Multiaccess channel, multiple keys, private key, private key capacity region, secrecy capacity, secret key, source emulation.

\section{INTRODUCTION}

$\mathbf{S}$ EPARATE terminals with the means to transact over a secure noisy channel as well as a public noiseless channel can devise a secret key more effectively than by using the secure channel alone. A secret key, in the Shannon theoretic sense, is common randomness of near uniform distribution regarding which an eavesdropper, that observes the public communication and perhaps also possesses additional observations available or unavailable to the terminals engaged in secrecy generation, can glean only a negligible amount of information.

The first Shannon theoretic model for generating a secret key over a noisy channel was Wyner's wiretap channel [24], generalized by Csiszár and Körner [7]. This model did not allow for public communication, and secret key generation was tantamount to secure transmission over the noisy channel, when the eavesdropper had access to wiretapped side information. The fact that secrecy generation could be enhanced by public communication was illustrated by Bennett et al. [3]. Models for secrecy generation, which entailed two terminals communicating over a public noiseless channel, were examined in detail by Maurer [17] and Ahlswede and Csiszár [1]. These models

Manuscript received September 02, 2011; revised July 05, 2012; accepted July 15, 2012. Date of publication September 04, 2012; date of current version December 19, 2012. I. Csiszár was supported by the Hungarian National Foundation for Scientific Research under Grant 76088. P. Narayan was supported by the U.S. National Science Foundation under Grants CCF0830697 and CCF1117546. This paper was presented in part at the 2009 IEEE International Symposium on Information Theory.

I. Csiszár is with the A. Rényi Institute of Mathematics, Hungarian Academy of Sciences, H-1364 Budapest, Hungary (e-mail: csiszar@renyi.hu).

P. Narayan is with the Department of Electrical and Computer Engineering and the Institute for Systems Research, University of Maryland, College Park, MD 20742 USA (e-mail: prakash@umd.edu).

Communicated by S. Diggavi, Associate Editor for Shannon Theory.

Digital Object Identifier 10.1109/TIT.2012.2216254 involve either a discrete memoryless multiple source (DMMS) with two components accessible to one terminal each, or a discrete memoryless channel (DMC) with one input terminal and one output terminal. In both types of models, an additional "wiretapped" terminal may or may not be present. The sizable literature on such models includes Maurer [18], Bennett, Brassard, Crépeau, and Maurer [4], Csiszár [6], Maurer and Wolf [19], [20], Csiszár and Narayan [9], [10], Renner and Wolf [21], Gohari and Anantharam [12], [13] and a comprehensive treatment in Csiszár and Körner [8]. A single-letter characterization of the secrecy capacity - the largest rate at which a secret key can be generated-is known in special cases, e.g., when a wiretapped terminal is absent or when the wiretapped terminal reveals itself to the parties generating secrecy.

In our previous work, we had studied secrecy generation for a multiterminal source model where each participating terminal had access to one component of a DMMS [9], [10], and for a multiterminal channel model which involved an underlying DMC with a single input and multiple outputs [10]; in both models, unrestricted and noiseless public communication between the terminals was permitted, to which the eavesdropper had full access. In this paper, which constitutes a continuation of our work in [10] and [11], we examine channel models for secrecy generation which involve an underlying DMC with multiple inputs and outputs. Terminals $1, \ldots, k$ govern the inputs and terminals $k+1, \ldots, m$ observe the corresponding outputs. Following each transmission of symbols by the input terminals over the DMC, communication over a public noiseless channel of unlimited capacity is allowed between all the terminals, which may be interactive and which is observed by all the terminals. ${ }^{1}$ The goal is to generate secret common randomness shared by a given set $A \subset\{1, \ldots, m\}$ of terminals at the largest rate possible. Thus, the resulting key must be accessible to every terminal in $A$. It need not be accessible to the terminals not in $A$, but nor is it required to be concealed from them, with the possible exception of a set $D$ of terminals which are "wiretapped" by the eavesdropper (where $A \cap D=\emptyset$ ). A DMC input terminal may or may not belong to the set $A$ or $D$.

We restrict ourselves to models where all the terminals cooperate, including those that are wiretapped (if $D \neq \emptyset$ ), in generating a secret key for the terminals in $A$, with secrecy being required from the eavesdropper that has access to only the public communication and the information available to the wiretapped terminals in $D$. Also, we assume the eavesdropper to be passive, i.e., unable to tamper with the communication of the legitimate terminals.

We do not address models with wiretap side information in which the underlying DMC also has an additional output terminal that is wiretapped by the eavesdropper and does not co-

${ }^{1}$ For ease of distinction between the use of the DMC and the use of the public channel, hereafter the former will be termed "transmission," while the latter will be referred to as "communication." 
operate in secrecy generation (cf., e.g., [1], [9], [10], [12], [13], [17], [19], and [21]).

The problem of secrecy generation for a general multiterminal channel model studied in this paper appears more difficult than its special case for a channel with a single input. Single-letter characterizations of secrecy capacities for the latter have been given in [10]. For the general channel model, short of providing single-letter characterizations of secrecy capacities, our main contributions are the following. One possible operational strategy in a channel model as above is source emulation which entails the channel input terminals (in the case when none is wiretapped) transmitting independent sequences of random variables (rvs) over the DMC with the output terminals observing the corresponding output sequences. In addition to this "simple" source emulation, we introduce also "general" source emulation that allows certain correlations among the rvs assigned to different input terminals even when none is wiretapped. The emulated source model leads to our achievability results which furnish lower bounds for the secrecy capacities in Theorem 4, using simple protocols. While our definition of general source emulation admits correlations between the input terminals, in the protocols that achieve our lower bounds, the input terminals operate independently of each other with each terminal transmitting independent sequences that need not be independent and identically distributed (i.i.d.). Our converse results provide upper bounds for the secrecy capacities using familiar techniques from Shannon theory, but are difficult and rely on two entropy inequalities from our previous work [10] which may be of independent interest. Our lower and upper bounds coincide only in special cases. While it is conceivable that our lower bounds could be always tight, the upper bounds are not so; evidence of the latter is shown by an improved upper bound for a class of channels.

We also consider multiterminal channel models in which different subsets $A_{i}$ of terminals share multiple keys, one for terminals in each set $A_{i}$ with secrecy required from the eavesdropper as well as from the terminals not in $A_{i}$. The main objective is to draw attention to the challenging problems in this realm that remain unresolved. Here, simple results are presented showing links among the associated secrecy capacity region for multiple keys, the transmission capacity region of the multiple access channel (MAC) defined by the DMC, and achievable rates for a single secret key shared by a subset of the terminals.

Our problem formulations are described in Section II. Section III treats secrecy generation for DMCs with a single output based on elementary considerations. Our general single-letter lower and upper bounds for secrecy capacities are presented in Sections IV and V, respectively. We illustrate our results and their limitations by four examples of secrecy generation in simple multiterminal channel models in Section VI. A closing discussion is contained in Section VII.

\section{PRELIMINARIES}

All rvs are assumed to take values in finite sets, even if not stated explicitly. An rv will be denoted by an uppercase letter and its range by the corresponding script capital unless stated otherwise. The cardinality of a finite set $\mathcal{X}$ is denoted by $|\mathcal{X}|$.
Logarithms are with respect to the base 2. For integers $l \leq k$, we denote $[l, k]=(l, \ldots, k)$.

We consider multiterminal channel models of the following kind. Terminals $1, \ldots, k$, with finite alphabets $\mathcal{X}_{1}, \ldots, \mathcal{X}_{k}$, are connected to terminals $k+1, \ldots, m$, with finite alphabets $\mathcal{X}_{k+1}, \ldots, \mathcal{X}_{m}$, respectively, by a DMC $W: \mathcal{X}_{1} \times \cdots \times \mathcal{X}_{k} \rightarrow \mathcal{X}_{k+1} \times \cdots \times \mathcal{X}_{m}$. Terminals $1, \ldots, k$ govern the inputs of the DMC over which they transmit securely sequences of length $n$, while terminals $k+1, \ldots, m$ observe the corresponding output sequences of length $n$. In between consecutive symbol transmissions over the DMC (with instantaneous receptions), the terminals in $\mathcal{M}=\{1, \ldots, m\}$ are allowed to communicate over a public noiseless channel of unlimited capacity. In any transmission or communication by a terminal, randomization is permitted. The public communication is observed by all the terminals in $\mathcal{M}$ as well as by an eavesdropper.

We shall assume that each terminal $i \in \mathcal{M}$ generates at the outset a rv $U_{i}$ to be used for randomization; the rvs $U_{1}, \ldots, U_{m}$ are mutually independent. Every input terminal $i \in[1, k]$ transmits $n$ symbols $X_{i 1}, X_{i 2}, \ldots, X_{i n}$ over the DMC $W$ at time instants $\tau_{1}<\tau_{2}<\cdots<\tau_{n}$, and every output terminal $i \in[k+1, m]$ observes the corresponding output symbols $X_{i 1}, X_{i 2}, \ldots, X_{i n}$. In addition, communication among the terminals in $\mathcal{M}$ over the public channel occurs-possibly interactively and in several rounds - during the time intervals $\left(\tau_{t}, \tau_{t+1}\right)$, for $t=1, \ldots, n-1$, and immediately following $\tau_{n}$, which hereafter will be referred to simply as intervals $t=1, \ldots, n$. The public communication of all the terminals in interval $t$ is depicted collectively as $F_{t}$, and we denote $\mathbf{F}=\left(F_{1}, \ldots, F_{n}\right)$.

In general, terminal $i \in[1, k]$ determines its $t$ th input $X_{i t}$ of the DMC $W$ as a function of $U_{i}$ for $t=1$, and of $\left(U_{i}, F_{1}, \ldots, F_{t-1}\right)$ for $t=2, \ldots, n$. Also, the communication of terminal $i \in \mathcal{M}$ in interval $t$ is allowed to depend on $U_{i}$, the symbols $\left(X_{i 1}, \ldots, X_{i t}\right)$ earlier generated or observed by terminal $i$, and on all earlier communication $\left(F_{1}, \ldots, F_{t-1}\right)$. While this general framework admits complex transmission and communication protocols, in our achievability proofs we shall use only simple noninteractive communication protocols with input terminals $i \in[1, k]$ not sending any public messages at all, and each output terminal $i \in[k+1, m]$ sending at most one public message $f_{i}=f_{i}\left(X_{i}^{n}\right)$ and that upon completion of the $n$ transmissions over the DMC; in this case, $\mathbf{F}=F_{n}=\left(f_{i}\left(X_{i}^{n}\right), i \in[1, k]\right)$.

For rvs $X_{i}, i \in \mathcal{M}$, we shall use the shorthand notation $X_{B}=\left(X_{i}, i \in B\right)$ for sets $B \subset \mathcal{M}$, and, as a special case, $X_{[a, b]}=\left(X_{a}, \ldots, X_{b}\right)$ for $1 \leq a \leq b \leq m$. Also, we shall write $X_{B}^{t}=\left(X_{B 1}, \ldots, X_{B t}\right)$ for $B \subset \mathcal{M}, 1 \leq t \leq n$, where $X_{B j}=\left(X_{i j}, i \in B\right), 1 \leq j \leq t$; in particular, $X_{i}^{t}=$ $\left(X_{i 1}, \ldots, X_{i t}\right)$ for $i \in \mathcal{M}, 1 \leq t \leq n$.

The following concepts introduced in [9] will be used. Given $\epsilon>0$, a rv $U$ is $\epsilon$-recoverable from $V$ if $\operatorname{Pr}\{U \neq f(V)\} \leq \epsilon$ for some function $f(V)$ of $V$. For rvs $K$ and $Y$, to be interpreted as representing a secret key and the eavesdropper's knowledge, respectively, the information theoretic security index is

$$
s(K ; Y)=\log |\mathcal{K}|-H(K \mid Y) .
$$


Smallness of this security index is tantamount jointly to a nearly uniform distribution for $K$ (i.e., $\log |\mathcal{K}|-H(K)$ is small) and to the near independence of $K$ and $Y$ (i.e., the mutual information $I(K \wedge Y)$ is close to 0$)$.

Definition 1: Given any set $A \subset \mathcal{M}$ of size $|A| \geq 2$, a rv $K$ constitutes an $(\epsilon, \delta)$-secret key $((\epsilon, \delta)$-SK) for the set of terminals $A$, achievable with $n$ uses of the DMC $W$, randomization $U_{\mathcal{M}}$ and public communication $\mathbf{F}$, if $K$ is $\epsilon$-recoverable from $\left(U_{i}, X_{i}^{n}, \mathbf{F}\right)$ for each $i \in A$ and, in addition, it satisfies the secrecy condition

$$
s(K ; \mathbf{F}) \leq \delta .
$$

An $(\epsilon, \delta)$-SK as earlier is called an $(\epsilon, \delta)$-private key $((\epsilon, \delta)$-PK) for the set of terminals $A$, private from the set of terminals $D \subset$ $\mathcal{M}$ with $A \cap D=\emptyset$, if it satisfies the stronger secrecy condition

$$
s\left(K ; U_{D}, X_{D}^{n}, \mathbf{F}\right) \leq \delta .
$$

By definition, an $(\epsilon, \delta)$-SK is recoverable at the terminals in $A$, and is nearly uniformly distributed and effectively concealed from an eavesdropper with access to the public communication $\mathbf{F}$; it need not be concealed from the terminals in $A^{c}=\mathcal{M} \backslash A$. On the other hand, an $(\epsilon, \delta)$-PK for $A$ is effectively concealed from an eavesdropper with access - in addition to the public communication $\mathbf{F}$-also to a set $D \subset A^{c}$ of "wiretapped" or "compromised" terminals. This $(\epsilon, \delta)$-PK need not be concealed from the terminals in $A^{c} \backslash D$. Note that the compromised terminals can cooperate in the secrecy generation through their public communication. Indeed, it can be assumed without loss of generality (w.l.o.g.) that the terminals in $D$ reveal publicly all the information in their possession (which, anyway, is accessible to the eavesdropper). This assumption will be made usually without explicit mention.

Definition 2: A number $R$ is an achievable SK rate for a set of terminals $A \subset \mathcal{M}$ if there exist $\left(\epsilon_{n}, \delta_{n}\right)$-secret keys $K^{(n)}$ achievable for $A$ with $n$ uses of the DMC $W$, suitable randomization $U_{\mathcal{M}}$, and public communication $\mathbf{F}^{(n)}$, such that

$$
\epsilon_{n} \rightarrow 0, \quad \delta_{n} \rightarrow 0 \quad \text { and } \quad \frac{1}{n} \log \left|\mathcal{K}^{(n)}\right| \rightarrow R \quad \text { as } \quad n \rightarrow \infty .
$$

The largest achievable SK rate for $A$ is the SK capacity $C_{S}(A)$. Achievable PK rates and PK capacity $C_{P}(A \mid D)$ are defined similarly.

Remark: Our converse proofs stand under the requirement of "weak" secrecy, i.e., $\delta_{n}=o(n)$ while $\epsilon_{n}$ decays to 0 [1], [17]. The achievability results hold with both $\epsilon_{n}$ and $\delta_{n}$ decaying to 0 exponentially rapidly thereby affording "strong" secrecy [6], [8], [18].

In general, any number of DMC input and output terminals may be wiretapped (barring two terminals to avoid the trivial). However, it is obvious that the wiretapped input terminals (if any) can be coalesced, as can the wiretapped output terminals. The next lemma shows that attention can be restricted even to such models in which no input terminal, and at most one output terminal, is wiretapped. Nevertheless, we shall find it convenient throughout to adhere to the original model above and take recourse only occasionally to the following reduction lemma.

Lemma 1: For any DMC $W: \mathcal{X}_{1} \times \cdots \times \mathcal{X}_{k} \rightarrow \mathcal{X}_{k+1} \times \cdots \times$ $\mathcal{X}_{m}$ and $D \subset \mathcal{M}$ with $0 \leq|D| \leq m-2$, there exists a DMC $\tilde{W}: \tilde{\mathcal{X}}_{1} \times \cdots \times \tilde{\mathcal{X}}_{\tilde{k}} \rightarrow \tilde{\mathcal{X}}_{k+1} \times \cdots \times \tilde{\mathcal{X}}_{\tilde{m}}$ with $\tilde{k}$ equal to the number of input terminals of $W$ not in $D$, or $\tilde{k}=1$ if $[1, k] \subset$ $D$, such that only terminal $\tilde{m}$ of $\tilde{W}$ is compromised, there is a bijection between the uncompromised terminals of $W$ and $\tilde{W}$, and for each $A \subset \mathcal{M}$ disjoint from $D$ the PK capacity $C_{P}(A \mid D)$ of $W$ is equal to the corresponding PK capacity $C_{P}(A \mid \tilde{D})$ of $\tilde{W}$ where $\tilde{D}=\{\tilde{m}\}$.

Remark: By the Lemma, any channel model with at most one uncompromised input terminal can be reduced to a model with just one input terminal; for the latter, a single-letter solution for PK capacity is available ([10, Th. 4.1]).

Proof: We can assume w.l.o.g. that $D=\{k, m\}$. Indeed, by the passage preceding the Lemma, it can be assumed that either $D=\{k\}$ or $D=\{k, m\}$, and in the former case, formally a compromised output terminal, can be added at which the output is a constant. Then, we shall prove the Lemma with $\tilde{m}=m$.

The compromised input terminal will be eliminated by merging it with an uncompromised one (if any, i.e., if $k>1$ ), while keeping track of it by letting the $m$-output of $\tilde{W}$ contain an identical copy of the compromised input of $W$.

Formally, assuming $D=\{k, m\}$, in case $k>1$ (when $\tilde{k}=$ $k-1)$, let $\tilde{\mathcal{X}}_{\tilde{k}} \triangleq \mathcal{X}_{k-1} \times \mathcal{X}_{k}, \tilde{\mathcal{X}}_{m}=\mathcal{X}_{m} \times \mathcal{X}_{k}$, and $\tilde{\mathcal{X}}_{i} \triangleq \mathcal{X}_{i}$ if $i \in[1, k-1] \cup[k+1, m-1]$. In case $k=1$ (when $\tilde{k}=1)$, the only modification needed is to set $\tilde{\mathcal{X}}_{\tilde{k}}=\tilde{\mathcal{X}}_{1} \triangleq \mathcal{X}_{1}$.

The input $\tilde{k}$-tuples of $\tilde{W}$ are identified in an obvious manner with input $k$-tuples $\mathbf{x}=x_{1}, \ldots, x_{k}$ of $W$, and output $(m-$ $k$ )-tuples of $\tilde{W}$ are regarded as being obtained by appending a symbol $x^{\prime}{ }_{k} \in \mathcal{X}_{k}$ to the output $(m-k)$-tuples $\mathbf{y}=x_{k+1} \ldots x_{m}$ of $W$. The definition of $\tilde{W}$ is

$$
\tilde{W}\left(\mathbf{y} x_{k}^{\prime} \mid \mathbf{x}\right) \triangleq \begin{cases}W(\mathbf{y} \mid \mathbf{x}) & \text { if } x^{\prime}{ }_{k}=x_{k} \\ 0 & \text { if } x^{\prime}{ }_{k} \neq x_{k}\end{cases}
$$

In other words, the DMC $\tilde{W}$ behaves as $W$ but additionally transmits noiselessly the input of terminal $k$ of $W$ to the terminal $\tilde{m}$ of $\tilde{W}$. Thus, the single wiretapped terminal $\tilde{m}$ of $\tilde{W}$ will possess the same information as the wiretapped terminals of $W$. It follows that each protocol for $W$ and $D$ gives rise to a protocol for $\tilde{W}$ and $\tilde{D}=\{\tilde{m}\}$ with identical secrecy performance, and also reciprocally so.

We shall also consider models in which different subsets of the terminals in $\mathcal{M}$ share multiple keys, one for the terminals in each subset with privacy from the remaining terminals in $\mathcal{M}$ that are not members of that subset.

Definition 3: Given different subsets $A_{1}, \ldots, A_{l}$ of $\mathcal{M}, l \geq$ 1 , the rvs $K_{1}, \ldots, K_{l}$ constitute $(\epsilon, \delta)$-PKs for the terminals in $A_{1}, \ldots, A_{l}$, respectively, if for each $i \in[1, l], K_{i}$ is an $(\epsilon, \delta)$-PK for the terminals in $A_{i}$, private from the terminals in $\mathcal{M} \backslash A_{i}$. The numbers $R_{1}, \ldots, R_{l}$ represent an achievable PK rate-tuple for 
the terminals in $A_{1}, \ldots, A_{l}$ if there exist $\left(\epsilon_{n}, \delta_{n}\right)$-PKs achievable for $A_{1}, \ldots, A_{l}$ with $n$ uses of the DMC $W$, suitable randomization $U_{\mathcal{M}}$, and public communication $\mathbf{F}^{(n)}$, such that

$$
\epsilon_{n} \rightarrow 0, \quad \delta_{n} \rightarrow 0 \quad \text { and } \quad \frac{1}{n} \log \left|\mathcal{K}_{i}^{(n)}\right| \rightarrow R_{i} \quad \text { as } \quad n \rightarrow \infty
$$

for $i=1, \ldots, l$. The set of all achievable PK rate-tuples is the PK capacity region $\mathcal{C}_{P}\left(A_{1}, \ldots, A_{l}\right)$. For the case $l=1$, $\mathcal{C}_{P}\left(A_{1}\right)=C_{P}\left(A_{1} \mid A_{1}^{c}\right)$ of Definition 2.

Remark: The PK capacity region is a closed convex set. The former is clear from the definition, while the latter is a consequence of a standard time-sharing argument.

\section{Models With Single OutPut}

In this section, we consider DMCs with a sole output for which simple results are presented that do not require any sophisticated tools.

Let $W: \mathcal{X}_{1} \times \cdots \times \mathcal{X}_{m-1} \rightarrow \mathcal{X}_{m}$ be a DMC with $k=m-1$ input terminals and one output terminal, and let $A$ be any set of terminals of size $|A| \geq 2$ which contains the output terminal $m$. Denote by $\mathcal{C}$ the (average error) capacity region of the MAC $W$, and by $\mathcal{C}(A)$ its projection on the $(|A|-1)$-dimensional subspace of $\mathbb{R}^{m-1}$ spanned by the coordinate axes $\{i: i \in A \backslash\{m\}\}$. Furthermore, consider the PK capacity region $\mathcal{C}_{P}\left(\left\{A_{i}, i \in A \backslash\{m\}\right\}\right)$ for the pairs of terminals $A_{i}=$ $\{i, m\}, i \in A \backslash\{m\}$.

Proposition 2: For $A$ and $A_{i}=\{i, m\}, i \in A \backslash\{m\}$ as earlier, it holds that

i) $\mathcal{C}_{P}\left(\left\{A_{i}, i \in A \backslash\{m\}\right\}\right) \supset \mathcal{C}(A)$;

ii) any $R>0$ such that the $(|A|-1)$-dimensional vector $(R, \ldots, R)$ belongs to $\mathcal{C}_{P}\left(\left\{A_{i}, i \in A \backslash\{m\}\right\}\right)$, is a lower bound for the PK capacity $C_{P}\left(A \mid A^{c}\right)$.

Corollary: It holds that

$$
\begin{aligned}
C_{S}(A) & \geq C_{P}\left(A \mid A^{c}\right) \\
& \geq \max \left\{R:(R, \ldots, R) \in \mathcal{C}_{P}\left(\left\{A_{i}, i \in A \backslash\{m\}\right\}\right)\right\} \\
& \geq \max \{R:(R, \ldots, R) \in \mathcal{C}(A)\} .
\end{aligned}
$$

Furthermore, any $R$ such that $(R, \ldots, R) \in \mathcal{C}(A)$ can be achieved as an SK rate for $A$ or PK rate for $A$ with privacy from $A^{c}$, with no public communication by the input terminals and with only the output terminal $m$ sending a public message.

\section{Proof:}

i) By definition, each $\left(R_{i}, i \in A \backslash\{m\}\right) \in \mathcal{C}(A)$ arises from some $\left(R_{1}, \ldots, R_{m-1}\right) \in \mathcal{C}$ by deleting the components $R_{i}$ with $i \notin A$; it can be supposed w.l.o.g. that all these deleted components are equal to 0 . It is easy to see that an achievable rate tuple $\left(R_{1}, \ldots, R_{m-1}\right)$ for transmission over a MAC $W$, in which some components $R_{i}$ are 0 , can be achieved by codes whose message sets corresponding to the zero rates are singletons (rather than merely of subexponential size). It follows that for each $\left(R_{i}, i \in A \backslash\{m\}\right) \in \mathcal{C}(A)$, there exist encoders $f_{i}: \mathcal{K}_{i} \rightarrow \mathcal{X}_{i}^{n}, i \in A \backslash\{m\}$, with $\left|\mathcal{K}_{i}\right|=\exp \left(n R_{i}^{\prime}\right)$, with $R_{i}^{\prime}$ arbitrarily close to $R_{i}$, and deterministic sequences $x_{i}^{n} \in \mathcal{X}_{i}^{n}, i \in[1, m-1] \backslash A$, with the following property: if the MAC inputs are

$$
X_{i}^{n} \triangleq \begin{cases}f_{i}\left(K_{i}\right), & i \in A \backslash\{m\} \\ x_{i}^{n}, & \text { otherwise }\end{cases}
$$

where the rvs $K_{i}$, uniformly distributed on $\mathcal{K}_{i}$, are mutually independent, then the rvs $K_{i}$ are recoverable from the MAC output $X_{m}^{n}$ with probability approaching 1 as $n \rightarrow 1$. This proves that $\left(R_{i}, i \in A \backslash\{m\}\right)$ is an achievable PK rate tuple for the pairs $A_{i}=\{i, m\}, i \in A \backslash\{m\}$, achievable without any public communication.

ii) Suppose that $(R, \ldots, R) \in \mathcal{C}_{P}\left(\left\{A_{i}, i \in A \backslash\{m\}\right\}\right)$, and consider PKs for the pairs $A_{i}=\{i, m\}, i \in A \backslash\{m\}$, represented by rvs $K_{i}$ distributed on $\mathcal{K} \triangleq\left\{1, \ldots, \exp \left(n R^{\prime}\right)\right\}$ with $R^{\prime}$ close to $R$, and satisfying the secrecy condition (2) with $\mathcal{M} \backslash\{i, m\}$ in the role of $D$. Then, arbitrarily fixing $i_{1} \in A \backslash\{m\}$, the rv $K_{i_{1}}$ becomes a PK for the terminals in $A$, private from $D \triangleq A^{c}$, if terminal $m$ broadcasts the $\bmod |\mathcal{K}|$ sums $K_{i_{1}}+K_{i}, i \in A \backslash\left\{i_{1}, m\right\}$.

The Corollary is immediate.

In Section VI, we shall give an example where the trivial inner bound for the PK capacity region $\mathcal{C}_{P}\left(\left\{A_{i}, i \in A \backslash\{m\}\right\}\right)$ and the lower bounds for the SK capacity $C_{S}(A)$, both above, are tight. It remains open whether they are tight in general. Here, we present a weaker result than the tightness of the lower bounds for $C_{S}(A)$ in the Corollary of Theorem 2, and which is straightforward.

Proposition 3: For any ${ }^{2} A \ni m$, the SK capacity $C_{S}(A)$ is positive iff there exists

$\left(R_{1}, \ldots, R_{m-1}\right) \in \mathcal{C}$ such that $R_{i}>0$ for each $i \in A \backslash\{m\}$.

Proof: Sufficiency is obvious by the Corollary of Proposition 2 . For necessity, note that if no $\left(R_{1}, \ldots, R_{m-1}\right)$ as above exists, then - using the convexity of $\mathcal{C}$ - for some $i_{1} \in A \backslash\{m\}$, we must have that $R_{i_{1}}=0$ for every $\left(R_{1}, \ldots, R_{m-1}\right) \in \mathcal{C}$. The latter means that $W\left(x_{m} \mid x_{1}, \ldots, x_{m-1}\right)$ does not depend on $x_{i_{1}}$, and this would imply that the SK capacity is 0 even if the terminals in $\mathcal{M} \backslash\left\{i_{1}\right\}$ were allowed to communicate securely among themselves. For a formal proof, note that upon regarding the terminals in $\mathcal{M} \backslash\left\{i_{1}\right\}$ as a consolidated party $L$, any use of the DMC $W$ amounts to a randomization performed by party $L$ (since the choice of channel input at terminal $i_{1}$ does not influence the output). However, it is well known that two parties (here $\left\{i_{1}\right\}$ and $L$ ), with no resources other than the ability of randomization and of public communication, cannot generate an SK; see, for example, [17].

Remark: Proposition 3 does not extend to DMCs with two or more outputs even if there is only one input. Indeed, for a DMC with a single input and $m-1 \geq 2$ outputs, the SK capacity can be positive even if each component channel $W_{i}, \quad i=2, \ldots, m-1$ (where $W_{i}\left(x_{i} \mid x_{1}\right)$ equals the sum of $W\left(x_{2}^{\prime}, \ldots, x_{m}^{\prime} \mid x_{1}\right)$ over all $x_{2}^{\prime}, \ldots, x_{m}^{\prime}$ with $\left.x_{i}^{\prime}=x_{i}\right)$ has capacity 0 ; see ([10, Example 1]). See also Example 4 in Section VI.

\footnotetext{
${ }^{2}$ The result holds also when $A \not \supset m$.
} 
We conclude this section by commenting on the possible relationship between SK capacity and the feedback capacity region $\mathcal{C}_{f}$ of a MAC, which may properly contain $\mathcal{C}$ [5]. Specifically, focusing for simplicity on the case $A=\mathcal{M}$, the Corollary of Proposition 2 suggests a comparison of the SK capacity $C_{S}=C_{S}(\mathcal{M})$ with

$$
R_{f}=\max \left\{R:(R, \ldots, R) \in \mathcal{C}_{f}\right\} .
$$

In the secrecy setting, full feedback is ruled out as the feedback communication is public. Still, if a coding scheme with partial feedback could be found by which the gain in transmission rates exceeds the information leakage due to feedback, it would lead to an SK rate larger than that in the Corollary of Proposition 2, even if it were less than $R_{f}$. The existence of such a scheme is unknown; the Cover-Leung scheme [5] does not appear to admit a modification with this property. While no evidence is available that $R_{f}$ might be an achievable SK rate, nor do we know, in general, even whether $C_{S} \leq R_{f}$. In Section V, the latter bound will be shown to hold for a class of MACs with two input terminals, for which a single-letter characterization of the feedback capacity region $\mathcal{C}_{f}$ is available.

\section{General Lower Bounds For SK AND PK CAPACITIES}

Our techniques developed in [10] will be used to derive bounds for SK and PK capacities for the general DMC model introduced in Section II. Our results are partial; unlike in [10], the lower bounds in this section and the upper bounds in the next section agree only in special cases.

One way to generate an SK or a PK for a multiterminal channel model is by simple source emulation. If the input terminals in $[1, k]$ use i.i.d. repetitions of a $k$-tuple of rvs $X_{1}, \ldots, X_{k}$, such that the $X_{i}$ s assigned to the nonwiretapped terminals $i \in[1, k] \backslash D$ are conditionally independent given $X_{[1, k] \cap D}$, the DMC $W$ will generate i.i.d. repetitions of an $m$-tuple of rvs $X_{1}, \ldots, X_{m}$, whose joint probability mass function (pmf) is given by

$$
\begin{aligned}
& P_{X_{\mathcal{M}}}\left(x_{[1, m]}\right) \\
& =P_{X_{[1, k]}}\left(x_{[1, k]}\right) W\left(x_{[k+1, m]} \mid x_{[1, k]}\right), \quad x_{[1, m]} \in \times_{i=1}^{m} \mathcal{X}_{i}
\end{aligned}
$$

with each output terminal $i \in[k+1, \ldots, m]$ observing i.i.d. repetitions of $X_{i}$. Clearly, achievable SK rates for the source model defined by $X_{\mathcal{M}}=\left(X_{1}, \ldots, X_{m}\right)$ will be achievable for the channel model, as well.

A general form of source emulation entails the use of an auxiliary source. Let us consider the PK generation problem with a given set $D \subset \mathcal{M}$ of wiretapped terminals; SK generation obtains as the special case $D=\emptyset$. Let $\mathcal{V}$ be a (finite) auxiliary alphabet, and consider rvs $V, X_{1}, \ldots, X_{k}$ such that $\left(V, X_{[1, k] \cap D}\right)$ has an arbitrary joint pmf, and the $X_{i} \mathrm{~s}, i \in[1, k] \backslash D$, are conditionally independent given $\left(V, X_{[1, k] \cap D}\right)$. Moreover, let $X_{i}, \quad i \in[k+1, m]$, represent the outputs of the DMC $W$ corresponding to input $X_{[1, k]}$, satisfying the Markov condition

$$
V-\circ-X_{[1, k]}-\circ-X_{[k+1, m]}
$$

so that the pmf of $\left(V, X_{\mathcal{M}}\right)$ is

$$
\begin{aligned}
& P_{V X_{\mathcal{M}}}\left(v, x_{[1, m]}\right) \\
& =P_{V X_{[1, k] \cap D}}(\tilde{v}) \times \\
& \times \prod_{i \in[1, k] \backslash D} P_{X_{i} \mid V X_{[1, k] \cap D}}\left(x_{i} \mid \tilde{v}\right) W\left(x_{[k+1, m]} \mid x_{[1, k]}\right)
\end{aligned}
$$

where $\tilde{v}=\left(v,\left\{x_{i}, i \in[1, k] \cap D\right\}\right)$.

An associated source model is defined by assigning rvs $V$ and $X_{i}, \quad i \in \mathcal{M}$, with a joint pmf as above, to $m+1$ terminals $0,1, \ldots, m$, letting the set of wiretapped terminals be $\bar{D} \triangleq D \cup\{0\}$. Clearly, this source model can be emulated by our given multiterminal channel model. First, the rvs $V, X_{[1, k] \cap D}$ with an arbitrarily specified joint pmf are generated by one of the input terminals and revealed as required by the source model (since $(\{0\} \cup D) \cap[1, k] \subset \bar{D})$. Then, the terminals $i \in[1, k] \backslash D$ can generate the rvs $X_{i}$ conditionally independently given $V, X_{[1, k] \cap D}$, and use them as their channel inputs while the rvs $X_{i}, i \in[1, k] \cap D$, are used as channel inputs by the corresponding terminals. These inputs, in turn, give rise to the channel outputs $X_{i}, i \in[k+1, m]$.

The single-letter formulas available for the SK and PK capacities of a source model [9], [10] afford lower bounds for the corresponding capacities of the multiterminal channel model, as the suprema of SK or PK capacities of source models obtainable by simple or general source emulation as above. These lower bounds will be stated formally in Theorem 4 .

As in [10], given any set $A \subset \mathcal{M}$ of size $|A| \geq 2$, we denote by $\mathcal{B}(A)$ the family of all nonempty sets $B \subset \mathcal{M}$ that do not contain $A$, and by $\Lambda(A)$ the set of all $|\mathcal{B}(A)|$-dimensional vectors $\lambda=\left\{\lambda_{B}: B \in \mathcal{B}(A)\right\}$, with $0 \leq \lambda_{B} \leq 1$, that satisfy

$$
\sum_{B \in \mathcal{B}(A): B \ni i} \lambda_{B}=1 \text { for each } i \in \mathcal{M} .
$$

Also, if a set $D \subset A^{c}$ is given, $\mathcal{B}(A \mid D)$ and $\Lambda(A \mid D)$ are defined analogously, restricting $B$ to subsets of $D^{c}$ and replacing (6) by

$$
\sum_{B \in \mathcal{B}(A \mid D): B \ni i} \lambda_{B}=1 \text { for each } i \in D^{c} .
$$

In the parlance of combinatorics, the vectors in $\Lambda(A)$ (resp. $\Lambda(A \mid D)$ ) are fractional partitions of $\mathcal{M}$ (resp. $D^{c}$ ) into members of $\mathcal{B}(A)$ (resp. $\mathcal{B}(A \mid D)$ ) (cf. e.g., [15]).

The following quantities will play an important role:

$$
G_{A}\left(X_{\mathcal{M}}, V, \lambda\right) \triangleq H\left(X_{\mathcal{M}} \mid V\right)-\sum_{B \in \mathcal{B}(A)} \lambda_{B} H\left(X_{B} \mid X_{B^{c}}, V\right)
$$

and

$$
\begin{aligned}
& G_{A \mid D}\left(X_{\mathcal{M}}, V, \lambda\right) \\
& \triangleq H\left(X_{\mathcal{M}} \mid X_{D}, V\right)-\sum_{B \in \mathcal{B}(A \mid D)} \lambda_{B} H\left(X_{B} \mid X_{B^{c}}, V\right)
\end{aligned}
$$

for rvs $X_{\mathcal{M}}, V$ (the latter with values in some finite set $\mathcal{V}$ ), and vectors $\lambda$ in $\Lambda(A)$ (resp. $\Lambda(A \mid D)$ ). We assume throughout, 
without further explicit mention, that the Markov condition (4) holds and that the pmf of $X_{\mathcal{M}}$ is compatible with the given DMC $W$, i.e.,

$$
\begin{aligned}
P_{V X_{\mathcal{M}}}\left(v, x_{[1, m]}\right)= & P_{V X_{[1, k]}}\left(v, x_{[1, k]}\right) W\left(x_{[k+1, m]} \mid x_{[1, k]}\right) \\
& v \in \mathcal{V}, x_{[1, m]} \in \times_{i=1}^{m} \mathcal{X}_{i}
\end{aligned}
$$

We denote by $G_{A}\left(X_{\mathcal{M}}, \lambda\right)$ and $G_{A \mid D}\left(X_{\mathcal{M}}, \lambda\right)$ the special cases for $V=$ constant of (8) and (9), respectively.

The quantities above are related to $G_{A}(Q, \lambda)$ and $G_{A \mid D}(Q, \lambda)$ defined in ([10, eqs. (6) and (7)]) for a DMC with a single input and $m$ outputs, with $Q$ denoting the input pmf. In order to apply the results of [10], we consider in the following an auxiliary channel model with underlying DMC $\bar{W}: \mathcal{V} \rightarrow \mathcal{X}_{1} \times \cdots \mathcal{X}_{m}$ (the input alphabet $\mathcal{V}$ being any finite set), which is defined by a DMC $W_{0}: \mathcal{V} \rightarrow \mathcal{X}_{1} \times \cdots \mathcal{X}_{k}$ as

$$
\begin{gathered}
\bar{W}\left(x_{[1, m]} \mid v\right) \triangleq W_{0}\left(x_{[1, k]} \mid v\right) W\left(x_{[k+1, m]} \mid x_{[1, k]}\right) \\
v \in \mathcal{V}, x_{\mathcal{M}} \in \times_{i=1}^{m} \mathcal{X}_{i} .
\end{gathered}
$$

Note that the sets $\mathcal{B}(A \mid D)$ and $\Lambda(A \mid D)$ corresponding to the original model (including when $D=\emptyset$ ) are the same as $\mathcal{B}(A \mid \bar{D})$ and $\Lambda(A \mid \bar{D})$ corresponding to the auxiliary model with $\bar{D} \triangleq D \cup$ $\{0\}$, where the fictitious terminal 0 depicts the input to the DMC $\bar{W}$ (as also $W_{0}$ ).

The rvs $V$ and $X_{\mathcal{M}}$ can be regarded, respectively, as the input and output of the DMC $\bar{W}$; in other words, they represent a source model that can be emulated by the auxiliary channel model iff their joint pmf is of the form (10) with

$$
\begin{aligned}
P_{V X_{[1, k]}}\left(v, x_{[1, k]}\right)= & P_{V}(v) W_{0}\left(x_{[1, k]} \mid v\right) \\
& v \in \mathcal{V}, x_{[1, k]} \in \times_{i=1}^{k} \mathcal{X}_{i} .
\end{aligned}
$$

This source model can be emulated by the original channel model iff the rvs $X_{i}, \quad i \in[1, k] \backslash D$ are conditionally independent given $V, X_{[1, k] \cap D}$. For rvs $V, X_{\mathcal{M}}$ satisfying (10) and (12), the quantities in (8) and (9) can be written equivalently in the notation of ([10, eqs. (6) and (7)]) as

$$
\begin{gathered}
G_{A}\left(X_{\mathcal{M}}, V, \lambda\right)=G_{A \mid \bar{D}}\left(P_{V}, \lambda\right), \quad \bar{D} \triangleq\{0\} \\
G_{A \mid D}\left(X_{\mathcal{M}}, V, \lambda\right)=G_{A \mid \bar{D}}\left(P_{V}, \lambda\right), \quad \bar{D} \triangleq D \cup\{0\}
\end{gathered}
$$

with the right sides meant for the underlying DMC $\bar{W}$. Hence, by [10, Th. 4.1], the minimum of $G_{A}\left(X_{\mathcal{M}}, V, \lambda\right)$ with respect to $\lambda \in \Lambda(A)$ is the PK capacity of the source model defined by $V, X_{\mathcal{M}}$, with privacy from terminal 0 ; furthermore, maximization over $P_{V}$ yields the PK capacity of the auxiliary channel model with underlying DMC $\bar{W}$. A similar statement holds for $G_{A \mid D}\left(X_{\mathcal{M}}, V, \lambda\right)$, with privacy from the terminals in $D \cup\{0\}$. Furthermore, by [10, Th. 4.2], for the auxiliary channel model, these PK rates are achievable by protocols such that terminal 0 transmits a deterministic sequence, and public communication takes place only after transmission over the DMC $\bar{W}$ has been completed and consists of public messages by the terminals in $[1, m]$ with at most one message from each terminal that is a (deterministic) function of the DMC outputs therein. Note that as a consequence of their operational meaning, the quantities in (13) and (14) are nonnegative.
Theorem 4: For any $V$, and $X_{1}, \ldots, X_{k}$ conditionally independent given $V$

$$
C_{S}(A) \geq \min _{\lambda \in \Lambda(A)} G_{A}\left(X_{\mathcal{M}}, V, \lambda\right)
$$

Similarly, for any $V$ and $X_{1}, \ldots, X_{k}$ such that $X_{i}, i \in[1, k] \cap$ $D^{c}$ are conditionally independent given $\left(V, X_{[1, k] \cap D}\right)$

$$
C_{P}(A \mid D) \geq \min _{\lambda \in \Lambda(A \mid D)} G_{A \mid D}\left(X_{\mathcal{M}}, V, \lambda\right) .
$$

Moreover, the right sides yield the largest SK or PK rates achievable by general source emulation using a particular choice of $V, X_{1}, \ldots, X_{k}$. These rates are achievable with a noninteractive communication protocol in which the input terminals operate independently of each other, with the terminals in $[1, k] \cap D$ transmitting deterministic sequences over the DMC $W$ and those in $[1, k] \backslash D$ transmitting independent sequences that are not necessarily i.i.d.

\section{Comments:}

1) The maxima of the right sides of (15) and (16) with respect to the choice of $V, X_{1}, \ldots, X_{k}$ are achieved since the cardinality of the range of $V$ can be bounded by standard techniques.

2) The largest SK or PK rates achievable by simple source emulation are obtained by a similar maximization of $G_{A}\left(X_{\mathcal{M}}, \lambda\right)$ or $G_{A \mid D}\left(X_{\mathcal{M}}, \lambda\right)$.

Proof: As discussed previously in Theorem 4, the righthand side of (15) is an achievable PK rate, in the auxiliary channel model with underlying DMC $\bar{W}$, for the set of terminals $A \subset \mathcal{M}$ with privacy from the input terminal 0 ; moreover, it is achievable by a protocol of the mentioned special kind that, in particular, has terminal 0 transmitting a deterministic sequence $v^{n}=\left(v_{1}, \ldots, v_{n}\right)$. The latter circumstance can be realized in the model with DMC $W$ with the input terminals simply transmitting mutually independent $\operatorname{rvs} X_{[1, k] t}, t=1, \ldots, n$, with pmfs $P_{X_{[1, k] t}}=P_{X_{[1, k]} \mid V=v_{t}}$, noting that it is at this point that the conditional independence hypothesis is used.

It follows, referring again to the preceding discussion, that the right-hand side of (15) is an achievable SK rate for the channel model with DMC $W$, by means of communication protocols admitting public communication only upon completion of the DMC transmissions and with each terminal $i \in \mathcal{M}$ sending at most one public message that is a function of $X_{i}^{n}$ alone. To complete the proof of Theorem 4 in respect of (15), it remains to show that the DMC input terminals $i \in[1, k]$ need not send public messages, to which end it may be necessary to change the pmfs of the input rvs $X_{i}^{n}$. This can be shown exactly as the analogous assertion of [10, Th. 2] was proved. Consider a "good" protocol in which the terminals $i \in[1, k]$ send public messages $f_{i}=f_{i}\left(X_{i}^{n}\right)$. Proceeding as in the cited proof (replacing $f_{0}$ there with $\left.\left(f_{1}, \ldots, f_{k}\right)\right)$, it follows that the protocol will remain "good" if the joint pmf of all $n$-length channel inputs is changed to its conditional joint pmf under the condition that the values of $f_{i}\left(X_{i}^{n}\right), i \in[1, k]$ are equal to suitable constants. This conditioning does not affect the independence of the inputs (although their components $X_{i t}, t=1, \ldots, n$, no longer need be independent), and it reduces the public messages $f_{i}\left(X_{i}^{n}\right)$ of the input terminals $i \in[1, k]$ to be constants. 
The assertion concerning (16) is proved in the same manner; this time, we define an auxiliary channel model with the role of $V$ assigned to $\left(V, X_{[1, k] \cap D}\right)$. It is obvious from the definition (9) of $G_{A \mid D}\left(X_{\mathcal{M}}, V, \lambda\right)$ that its value remains unchanged if $V$ is replaced by $\left(V, X_{[1, k] \cap D}\right)$.

Next, restricting attention to a MAC with a single output whose capacity region is $\mathcal{C}$, by the Corollary of Theorem 2 , the condition $(R, \ldots, R) \in \mathcal{C}$ is sufficient for $R$ to be an achievable SK rate for $A=\mathcal{M}$. While it remains unclear whether this condition is necessary, the next Proposition shows that larger SK rates cannot be achieved by means of general source emulation.

Proposition 5: For a MAC $W: \mathcal{X}_{1} \times \cdots \times \mathcal{X}_{m-1} \rightarrow$ $\mathcal{X}_{m}$, a necessary and sufficient condition for the achievability of SK rate $R$ with $A=\mathcal{M}$ by general source emulation is $(R, \ldots, R) \in \mathcal{C}$.

Comment: A similar argument shows that $R$ is achievable as an SK rate by simple source emulation iff $(R, \ldots, R)$ belongs to a polyhedron

$$
\begin{aligned}
& \left\{\left(R_{1}, \ldots, R_{m-1}\right): R_{i} \geq 0,\right. \\
& \left.\sum_{i \in B} R_{i} \leq I\left(X_{B} \wedge X_{m} \mid X_{B^{c} \backslash\{m\}}\right), B \subset[1, m-1]\right\}
\end{aligned}
$$

where $X_{1}, \ldots, X_{m-1}$ are i.i.d. rvs and $P_{X_{m} \mid X_{[1, m-1]}}=W$. Since the capacity region $\mathcal{C}$ equals the convex closure of the union of all such polyhedra, where the union itself may be nonconvex, this shows that for some MACs, general source emulation can yield larger SK rates than simple source emulation; see Example 3 in Section VI as follows.

Proof: Consider general source emulation involving an auxiliary rv $V$ and input rvs $X_{1}, \ldots X_{m-1}$ that are conditionally independent given $V$, and let $X_{m}$ be the corresponding output rv. By Theorem 4, the SK rate achievable by this source emulation is $\min _{\lambda \in \Lambda(\mathcal{M})} G_{\mathcal{M}}\left(X_{\mathcal{M}}, V, \lambda\right)$.

Since $X_{1}, \ldots, X_{m-1}$ are conditionally independent given $V$, and $V-\circ-X_{[1, m-1]}-\circ-X_{m}$, the expression for $G_{\mathcal{M}}\left(X_{\mathcal{M}}, V, \lambda\right)$ in (8) simplifies. Specifically

$$
\begin{aligned}
H\left(X_{\mathcal{M}} \mid V\right) & =H\left(X_{[1, m-1]} \mid V\right)+H\left(X_{m} \mid X_{[1, m-1]}, V\right) \\
& =\sum_{i=1}^{m-1} H\left(X_{i} \mid V\right)+H\left(X_{m} \mid X_{[1, m-1]}\right)
\end{aligned}
$$

for $B \ni m$

$$
\begin{aligned}
H\left(X_{B} \mid X_{B^{c}}, V\right) & \\
& =H\left(X_{B \backslash\{m\}}, X_{m} \mid X_{B^{c}}, V\right) \\
& =H\left(X_{B \backslash\{m\}} \mid X_{B^{c}}, V\right)+H\left(X_{m} \mid X_{[1, m-1]}, V\right) \\
& =\sum_{i \in B \backslash\{m\}} H\left(X_{i} \mid V\right)+H\left(X_{m} \mid X_{[1, m-1]}\right)
\end{aligned}
$$

and for $B \not \supset m$

$$
\begin{aligned}
& H\left(X_{B} \mid X_{B^{c}}, V\right) \\
= & H\left(X_{B} \mid X_{B^{c}} \backslash\{m\}, V\right)-I\left(X_{B} \wedge X_{m} \mid X_{B^{c} \backslash\{m\}}, V\right) \\
= & \sum_{i \in B} H\left(X_{i} \mid V\right)-I\left(X_{B} \wedge X_{m} \mid X_{B^{c} \backslash\{m\}}, V\right) .
\end{aligned}
$$

Substituting (17)-(19) into (8), and using $\sum_{B: B \ni i} \lambda_{B}=1$ for each $i \in \mathcal{M}$, we obtain

$$
G_{\mathcal{M}}\left(X_{\mathcal{M}}, V, \lambda\right)=\sum_{B \in \Lambda(\mathcal{M}): B \not \supset m} \lambda_{B} I\left(X_{B} \wedge X_{m} \mid X_{B^{c} \backslash\{m\}}, V\right) .
$$

For any fixed $\tilde{B} \subset[1, m-1]$, assign $\lambda \in \Lambda(\mathcal{M})$ defined by $\lambda_{B}=\frac{1}{|\tilde{B}|}$ if $B=\tilde{B}$ or $B=\mathcal{M} \backslash\{i\}$ for some $i \in \tilde{B}$, and $\lambda_{B}=0$ otherwise. With this $\lambda$, (20) gives

$$
G_{\mathcal{M}}\left(X_{\mathcal{M}}, V, \lambda\right)=\frac{1}{|\tilde{B}|} I\left(X_{\tilde{B}} \wedge X_{m} \mid X_{\tilde{B}^{c} \backslash\{m\}}, V\right) .
$$

It follows that $R=\min _{\lambda \in \Lambda(\mathcal{M})} G_{\mathcal{M}}\left(X_{\mathcal{M}}, V, \lambda\right)$ satisfies

$$
R|\tilde{B}| \leq I\left(X_{\tilde{B}} \wedge X_{m} \mid X_{\tilde{B}^{c} \backslash\{m\}}, V\right)
$$

for every $\tilde{B} \subset[1, m-1]$, proving the necessity part of the assertion.

For sufficiency, note that $(R, \ldots, R) \in \mathcal{C}$ means that for some $V$ and $X_{1}, \ldots, X_{m-1}$ conditionally independent given $V$ with $V-\circ-X_{[1, m-1]}-\circ-X_{m}$, the inequalities (21) are satisfied. For these rvs, (20) and (21) give

$$
\begin{aligned}
& G_{\mathcal{M}}\left(X_{\mathcal{M}}, V, \lambda\right) \geq \sum_{B \in \Lambda(\mathcal{M}): B \not m m} \lambda_{B} R|B| \\
& \geq R\left(\sum_{B \in \Lambda(\mathcal{M})} \lambda_{B}|B|-\sum_{B \in \Lambda(\mathcal{M}): B \ni m} \lambda_{B}(m-1)\right) .
\end{aligned}
$$

Since $\sum_{B \in \Lambda(\mathcal{M})} \lambda_{B}|B|=\sum_{i=1}^{m} \sum_{B \in \Lambda(\mathcal{M}): B \ni i} \lambda_{B}=m$ and $\sum_{B \in \Lambda(\mathcal{M}): B \ni m} \lambda_{B}=1$, this proves that $\min _{\lambda \in \Lambda(\mathcal{M})} G_{\mathcal{M}}\left(X_{\mathcal{M}}, V, \lambda\right) \geq R$.

\section{GeNERAL UpPer Bounds FOR SK AND PK CAPACITIES}

In order to state our upper bounds for $C_{S}(A)$ and $C_{P}(A \mid D)$, we extend the notation in (8) and (9) above with a slight abuse of it. Specifically, for rvs $X_{\mathcal{M}}, V$, and for $\lambda \in \Lambda(A)$ or $\lambda \in$ $\Lambda(A \mid D)$, we denote

$$
\begin{aligned}
& G_{A}\left(X_{[1, k]}, V, \lambda\right) \triangleq H\left(X_{[1, k]} \mid V\right) \\
& -\sum_{B \in \mathcal{B}(A)} \lambda_{B} H\left(X_{[1, k] \cap B} \mid X_{[1, k] \cap B^{c}}, V\right) \\
& G_{A \mid D}\left(X_{[1, k]}, V, \lambda\right) \triangleq H\left(X_{[1, k]} \mid X_{D}, V\right) \\
& -\sum_{B \in \mathcal{B}(A \mid D)} \lambda_{B} H\left(X_{[1, k] \cap B} \mid X_{[1, k] \cap B^{c}}, X_{D}, V\right)
\end{aligned}
$$

and denote by $G_{A}\left(X_{[1, k]}, \lambda\right)$ and $G_{A \mid D}\left(X_{[1, k]}, \lambda\right)$ the special cases for $V=$ constant of (22) and (23), respectively. As earlier, we assume that the rvs $V, X_{\mathcal{M}}$ satisfy the Markov condition $V-0-X_{[1, k]}-0-X_{[k+1, m]}$ and $P_{X_{[k+1, m]}{ }^{\prime} X_{[1, k]}}=W$. Akin to $G_{A}\left(X_{\mathcal{M}}, V, \lambda\right)$ and $G_{A \mid D}\left(X_{\mathcal{M}}, V, \lambda\right)$ in (13) and (14), both $G_{A}\left(X_{[1, k]}, V, \lambda\right)$ and $G_{A \mid D}\left(X_{[1, k]}, V, \lambda\right)$, too, are nonnegative, as shown in Appendix B.

Theorem 6: The SK capacity $C_{S}(A)$ for a set of terminals $A \subset \mathcal{M}$ and the PK capacity $C_{P}(A \mid D)$ for $A$ with privacy from 
a set of terminals $D \subset A^{c}$ are bounded above, respectively, as follows:

$$
C_{S}(A) \leq \sup _{P_{V, X_{[1, k]}}} \inf _{\lambda \in \Lambda(A)}\left[G_{A}\left(X_{\mathcal{M}}, V, \lambda\right)-G_{A}\left(X_{[1, k]}, V, \lambda\right)\right]
$$

and for any $i \in D^{c}$

$$
\begin{aligned}
C_{P}(A \mid D) \leq \sup _{P_{V, X_{[1, k]}}} \inf _{\lambda \in \Lambda(A \mid D)}\left[G_{A \mid D}\left(X_{\mathcal{M}}, V, \lambda\right)\right. \\
-G_{A \mid D}\left(X_{[1, k]}, V, \lambda\right) \\
\left.+\sum_{B \in \mathcal{B}(A \mid D): B \not \supset i} \lambda_{B} I\left(X_{D} \wedge X_{[1, k] \cap B} \mid X_{[1, k] \cap B^{c}}, V\right)\right] .
\end{aligned}
$$

Furthermore, in the single output case $k=m-1$, provided that the output is not compromised, i.e., $D \not \supset m$

$$
\begin{aligned}
C_{P}(A \mid D) \leq & \sup _{P_{V, X_{[1, m-1]}}} \inf _{\lambda \in \Lambda(A \mid D)} \sum_{B \in \mathcal{B}(A \mid D): B \not \supset m} \lambda_{B} \times \\
& \times I\left(X_{m} \wedge X_{B} \mid X_{[1, m-1] \cap B^{c}}, V\right) .
\end{aligned}
$$

Corollary: When all the DMC inputs except perhaps one are compromised

$$
C_{P}(A \mid D)=\sup _{P_{V, X_{[1, k]}}} \inf _{\lambda \in \Lambda(A \mid D)} G_{A \mid D}\left(X_{\mathcal{M}}, V, \lambda\right) .
$$

\section{Comments:}

1) If $D \subset[1, k]$, then the last term in (25) vanishes. If $D \supset$ $[k+1, \ldots, m]$, then the difference of the first two terms is 0.

2) The Corollary is a consequence also of the Remark following Lemma 1.

3) The hypothesis of the Corollary is fulfilled when the DMC has only one input terminal. In this case, the Corollary reduces to [10, Th. 4.1].

4) The upper bound in (24) can be weakened to $C_{S}(A) \leq$ $\sup _{\left.P_{V, X}, k\right]} \inf _{\lambda \in \Lambda(A)} \quad G_{A}\left(X_{\mathcal{M}}, V, \lambda\right)$. This weaker bound differs from the lower bound in Theorem 4 by the lack of the conditional independence of $X_{1}, \ldots, X_{k}$ given $V$. It remains open whether (25) can be similarly weakened.

Proof: The upper bound for PK capacity in (25) is derived first. The bound in (24) for SK capacity follows as the special case $D=\emptyset$.

The initial steps in proving (25) are identical to those in the proof of the analogous converse part in [10, Th. 4.1]. These steps are presented first in a summarized form below, which then serve as a point of departure for the rest of the proof.

Suppose that the rv $K^{(n)}$ represents an $\left(\epsilon_{n}, \delta_{n}\right)$-PK with privacy from $D \subset A^{c}$, achievable with randomization $U_{\mathcal{M}}$ and public communication $\mathbf{F}^{(n)}$, where $\delta_{n}=o(n)$ and $\epsilon_{n} \rightarrow 0$; see Definition 2 and the succeeding remark. As observed in [10, the remark preceding Definition 3], we can suppose w.l.o.g. that
$\mathbf{F}^{(n)}=\left(U_{D}, X_{D}^{n}, \tilde{\mathbf{F}}^{(n)}\right)$, where $\tilde{\mathbf{F}}^{(n)}$ consists of the communication of all the terminals in $D^{c}$. Then, the secrecy condition (2) is

$$
\log \left|\mathcal{K}^{(n)}\right|-H\left(K^{(n)} \mid U_{D}, X_{D}^{n}, \tilde{\mathbf{F}}^{(n)}\right) \leq \delta_{n}
$$

Using [10, Appendix A, Corollary of Lemma A.2] with $\left(U_{i}, X_{i}^{n}\right)$ in the role of $X_{i}, i \in \mathcal{M}$, and $X_{D}^{n}$ and $\tilde{\mathbf{F}}^{(n)}$ in the roles of $X_{D}$ and $Y$, respectively, we get as in [10, inequality (11)] that for every $\lambda=\left\{\lambda_{B}: B \in \mathcal{B}(A \mid D)\right\} \in \Lambda(A \mid D)$

$$
\begin{gathered}
\frac{1}{n} \log \left|\mathcal{K}^{(n)}\right| \leq \frac{\alpha_{n}}{n}\left[\left\{H\left(U_{\mathcal{M}}, X_{\mathcal{M}}^{n} \mid U_{D}, X_{D}^{n}, \tilde{\mathbf{F}}^{(n)}\right)\right.\right. \\
\left.\left.-\sum_{B \in \mathcal{B}(A \mid D)} \lambda_{B} H\left(U_{B}, X_{B}^{n} \mid U_{B^{c}}, X_{B^{c}}^{n}, \tilde{\mathbf{F}}^{(n)}\right)\right\}\right]+\beta_{n}
\end{gathered}
$$

where

$$
\alpha_{n} \rightarrow 1 \quad \text { and } \quad \beta_{n} \rightarrow 0 \quad \text { as } \quad n \rightarrow \infty .
$$

A main ingredient of the proof of (25) will be to show that the expression within $[\cdots]$ above is bounded above by

$$
\begin{aligned}
& \sum_{t=1}^{n}\left[\left(H\left(X_{\mathcal{M} t} \mid X_{D t}\right)-\sum_{B \in \mathcal{B}(A \mid D)} \lambda_{B} H\left(X_{B t} \mid X_{B^{c} t}\right)\right)\right. \\
& -\left(H\left(X_{([1, k]) t} \mid X_{D t}\right)\right. \\
& \left.-\sum_{B \in \mathcal{B}(A \mid D)} \lambda_{B} H\left(X_{([1, k] \cap B) t} \mid X_{\left([1, k] \cap B^{c}\right) t}, X_{D t}\right)\right) \\
& \left.+\sum_{B \in \mathcal{B}(A \mid D): B \nexists i} \lambda_{B} I\left(X_{D t} \wedge X_{([1, k] \cap B) t} \mid X_{\left([1, k] \cap B^{c}\right) t}\right)\right]
\end{aligned}
$$

for $i \in D^{c}$. This implication is a generalization of the fact proved in [10] that the bracketed expression in (11) therein was bounded above by (12). Accordingly, the proof of the claimed implication here is similar to, but more complicated than, the corresponding proof in [10]. This proof, provided in Appendix A, required a new idea in establishing (A8).

To simplify (29), a standard technique is used: Let $V$ be an auxiliary rv distributed uniformly on $\{1, \ldots, n\}$ and independent of $X_{\mathcal{M}}^{n}$, and set $\tilde{X}_{i} \triangleq X_{i V}, \quad i \in \mathcal{M}$. Then, $\sum_{t=1}^{n} H\left(X_{\mathcal{M} t} \mid X_{D t}\right)=n H\left(\tilde{X}_{\mathcal{M}} \mid \tilde{X}_{D}, V\right)$, etc., and it holds that $V-\circ-\tilde{X}_{[1, k]}-\circ-\tilde{X}_{[k+1, m]}$ and $P_{\tilde{X}_{[k+1, m]} \mid \tilde{X}_{[1, k]}}=W$. Finally, omitting the tildes, we obtain for the new $X_{\mathcal{M}}$ from (28), (29) (and recalling (9), (23)) that

$$
\begin{aligned}
& \limsup \frac{1}{n} \log \left|\mathcal{K}^{(n)}\right| \leq G_{A \mid D}\left(X_{\mathcal{M}}, V, \lambda\right) \\
& -G_{A \mid D}\left(X_{[1, k]}, V, \lambda\right) \\
& +\sum_{B \in \mathcal{B}(A \mid D): B \not \supset i} \lambda_{B} I\left(X_{D} \wedge X_{[1, k] \cap B} \mid X_{[1, k] \cap B^{c}}, V\right)
\end{aligned}
$$

for every $\lambda=\left\{\lambda_{B}: B \in \mathcal{B}(A \mid D)\right\} \in \Lambda(A \mid D)$ and $i \in D^{c}$. The claimed upper bound for PK rates in (25) follows thereupon. 
Turning to the special case $k=m-1$ and $D \not \nexists m$, note that the last term in (25) vanishes. For $B \in \mathcal{B}(A \mid D)$, since $[1, m-1] \cap B^{c} \supset D$, we have in (25), by (9) and (23), that

$$
\begin{aligned}
& G_{A \mid D}\left(X_{\mathcal{M}}, V, \lambda\right)-G_{A \mid D}\left(X_{[1, m-1]}, V, \lambda\right) \\
&=\left(H\left(X_{\mathcal{M}} \mid X_{D}, V\right)-\sum_{B \in \mathcal{B}(A \mid D)} \lambda_{B} H\left(X_{B} \mid X_{B^{c}}, V\right)\right) \\
&-\left(H\left(X_{[1, m-1]} \mid X_{D}, V\right)\right. \\
&-\left.\sum_{B \in \mathcal{B}(A \mid D)} \lambda_{B} H\left(X_{[1, m-1] \cap B} \mid X_{[1, m-1] \cap B^{c}}, V\right)\right) \\
&= H\left(X_{m} \mid X_{[1, m-1]}\right) \\
&- \sum_{B \in \mathcal{B}(A \mid D)} \lambda_{B}\left(H\left(X_{B} \mid X_{B^{c}}, V\right)\right. \\
&-\left.H\left(X_{[1, m-1] \cap B} \mid X_{[1, m-1] \cap B^{c}}, V\right)\right) \\
&= H\left(X_{m} \mid X_{[1, m-1]}\right) \\
&- \sum \lambda_{B}\left(H\left(X_{[1, m-1] \cap B} \mid X_{B^{c}}, V\right)\right. \\
&+ H\left(X_{B \backslash[1, m)}\right. \\
&-\left.H\left(X_{[1, m-1] \cap B} \mid X_{[1, m-1] \cap B^{c}}, V\right)\right) .
\end{aligned}
$$

Now, for $m \in B$, in the summand in the right-hand side of (31), the expression within $(\cdots)$ equals

$$
\begin{aligned}
& H\left(X_{[1, m-1] \cap B} \mid X_{B^{c}}, V\right)+H\left(X_{m} \mid X_{[1, m-1]}\right) \\
& -H\left(X_{[1, m-1] \cap B} \mid X_{B^{c}}, V\right)=H\left(X_{m} \mid X_{[1, m-1]}\right)
\end{aligned}
$$

while for $m \notin B$, it equals

$$
\begin{aligned}
& H\left(X_{[1, m-1] \cap B} \mid X_{[1, m-1] \cap B^{c}}, X_{m}, V\right)+0 \\
& -H\left(X_{[1, m-1] \cap B} \mid X_{[1, m-1] \cap B^{c}}, V\right) \\
& =I\left(X_{m} \wedge X_{B} \mid X_{[1, m-1] \cap B^{c}}, V\right) .
\end{aligned}
$$

By (32), (33), and using $\sum_{B \in \mathcal{B}(A \mid D): B \ni m} \lambda_{B}=$ 1 , it follows that the right-hand side of (31) equals $\sum_{B \in \mathcal{B}(A \mid D): B \not \supset m} \lambda_{B} I\left(X_{m} \wedge X_{B} \mid X_{[1, m-1] \cap B^{c}}, V\right)$, thereby leading to (26).

Proof of Corollary: Under the hypothesis of the Corollary, $G_{A \mid D}\left(X_{[1, k]}, V, \lambda\right)=0$, and the last term in (25) also vanishes, trivially when $D \supset[1, k]$, and upon taking $i$ to be the uncompromised input otherwise. Hence, in this case, (25) gives the upper bound

$$
C_{P}(A \mid D) \leq \sup _{P_{V, x_{[1, k]}}} \inf _{\lambda \in \Lambda(A \mid D)} G_{A \mid D}\left(X_{\mathcal{M}}, V, \lambda\right)
$$

which coincides with the lower bound in Theorem 4 .

The upper bounds in Theorem 6 are not tight, in general, but only in special cases do refinements in the proof seem to yield improvements of interest. This is illustrated by the next theorem where the bound $C_{S} \leq R_{f}$ mentioned in Section III is established for a class of MACs with two inputs and one output. In the remainder of this section, $A=\mathcal{M}=\{1,2,3\}$, and $C_{S}(\mathcal{M})$ is denoted by $C_{S}$.
Consider the Willems' class $\mathcal{W}$ of two-input, one-output MACs with the property that input 1 is determined uniquely by input 2 and the output, i.e., there exists a mapping $\phi: \mathcal{X}_{2} \times \mathcal{X}_{3} \rightarrow \mathcal{X}_{1}$ such that $W\left(x_{3} \mid x_{1}, x_{2}\right)=0$ if $x_{1} \neq \phi\left(x_{2}, x_{3}\right)$. For this class of MACs, Willems [22] proved that the capacity region with feedback is equal to the Cover-Leung region [5]. Thus, for a MAC in class $\mathcal{W}$, $\left(R_{1}, R_{2}\right) \in \mathcal{C}_{f}$ if and only if there exist rvs $V, X_{1}, X_{2}, X_{3}$ whose joint pmf factorizes as

$$
\begin{aligned}
& P_{V X_{1} X_{2} X_{3}}\left(v, x_{1}, x_{2}, x_{3}\right) \\
& =P_{V}(v) P_{X_{1} \mid V}\left(x_{1} \mid v\right) P_{X_{2} \mid V}\left(x_{2} \mid v\right) W\left(x_{3} \mid x_{1}, x_{2}\right)
\end{aligned}
$$

and

$$
\begin{aligned}
& 0 \leq R_{1} \leq I\left(X_{1} \wedge X_{3} \mid X_{2}, V\right), 0 \leq R_{2} \leq I\left(X_{2} \wedge X_{3} \mid X_{1}, V\right) \\
& R_{1}+R_{2} \leq I\left(X_{1}, X_{2} \wedge X_{3}\right) .
\end{aligned}
$$

Consequently, the largest $R$ with $(R, R) \in \mathcal{C}_{f}$ is given by

$$
\begin{gathered}
R_{f}=\max \min \left\{I\left(X_{1} \wedge X_{3} \mid X_{2}, V\right), I\left(X_{2} \wedge X_{3} \mid X_{1}, V\right),\right. \\
\left.0.5 I\left(X_{1}, X_{2} \wedge X_{3}\right)\right\}
\end{gathered}
$$

with the maximum taken over rvs satisfying (34). Note that the range of the rv $V$ can be assumed to have a specified cardinality, and so the maximum in (35) is attained.

Theorem 7: For a MAC in class $\mathcal{W}$, it holds that $C_{S} \leq R_{f}$.

Proof: A slight refinement of the proof of Theorem 6, specialized to the case $A=\mathcal{M}=\{1,2,3\}$, shows that the expression within $[\ldots]$ in (28) now is bounded above by

$$
\begin{aligned}
\sum_{t=1}^{n} & {\left[\lambda_{\{1\}} I\left(X_{1 t} \wedge X_{3 t} \mid X_{2 t},\left(X_{1}, X_{3}\right)^{t-1}, F^{t-1}, U_{3}\right)\right.} \\
& +\lambda_{\{2\}} I\left(X_{2 t} \wedge X_{3 t} \mid X_{1 t},\left(X_{1}, X_{3}\right)^{t-1}, F^{t-1}, U_{3}\right) \\
& \left.+\lambda_{\{1,2\}} I\left(X_{1 t}, X_{2 t} \wedge X_{3 t} \mid X_{3}^{t-1}, F^{t-1}, U_{3}\right)\right] .
\end{aligned}
$$

See (A15) in Appendix A. In particular, the terms corresponding to the sets $B \in \mathcal{B}(\mathcal{M})$ that contain the output terminal 3 are equal to 0 . As in the proof of Theorem 6, this sum can be rewritten as

$$
\begin{aligned}
& n\left[\lambda_{\{1\}} I\left(X_{1} \wedge X_{3} \mid X_{2}, V\right)+\lambda_{\{2\}} I\left(X_{2} \wedge X_{3} \mid X_{1}, V\right)\right. \\
& \left.\quad+\lambda_{\{1,2\}} I\left(X_{1}, X_{2} \wedge X_{3} \mid V_{0}\right)\right]
\end{aligned}
$$

where

$$
\begin{array}{r}
V_{0} \triangleq X_{3}^{T-1}, F^{T-1}, U_{3}, T, V \triangleq V_{0}, X_{1}^{T-1}, X_{i} \triangleq X_{i T}, \\
i=1,2,3
\end{array}
$$

with $T$ being distributed uniformly on $\{1, \ldots, n\}$. Then the Markov relation $V_{0}-0-V-0-X_{1}, X_{2}-0-X_{3}$ holds and, 
moreover, $X_{1}$ and $X_{2}$ are conditionally independent given $V$. The latter follows by the dependence balance bound technique of Hekstra and Willems [14]. Indeed, Lemma C in Appendix C implies that

$$
\begin{aligned}
& I\left(U_{1} \wedge U_{2} \mid\left(X_{1}, X_{3}\right)^{n}, F^{n}, U_{3}\right) \\
& \leq-\sum_{t=1}^{n} I\left(X_{1 t} \wedge X_{2 t} \mid\left(X_{1}, X_{3}\right)^{t-1}, F^{t-1}, U_{3}\right) \\
& =-n I\left(X_{1} \wedge X_{2} \mid V\right) .
\end{aligned}
$$

Hence, using the trivial bound $I\left(X_{1}, X_{2} \wedge X_{3} \mid V_{0}\right) \leq$ $I\left(X_{1}, X_{2} \wedge X_{3}\right)$, it follows from (28) that the maximum, subject to (34), of

$$
\begin{aligned}
\min _{\lambda \in \Lambda(\mathcal{M})}[ & \lambda_{\{1\}} I\left(X_{1} \wedge X_{3} \mid X_{2}, V\right)+\lambda_{\{2\}} I\left(X_{2} \wedge X_{3} \mid X_{1}, V\right) \\
& \left.+\lambda_{\{1,2\}} I\left(X_{1}, X_{2} \wedge X_{3}\right)\right]
\end{aligned}
$$

is an upper bound for $C_{S}$.

Finally, for the existence of $\lambda \in \Lambda(\mathcal{M})$ with specified $\lambda_{\{1\}} \geq$ $0, \lambda_{\{2\}} \geq 0, \lambda_{\{1,2\}} \geq 0$, the inequalities

$$
\begin{aligned}
& \lambda_{\{1\}}+\lambda_{\{1,2\}} \leq 1, \lambda_{\{2\}}+\lambda_{\{1,2\}} \leq 1 \\
& \lambda_{\{1\}}+\lambda_{\{2\}}+2 \lambda_{\{1,2\}} \geq 1
\end{aligned}
$$

are necessary and sufficient. Subject to these conditions, the minimum above is attained when two of $\lambda_{\{1\}}, \lambda_{\{2\}}, \lambda_{\{1,2\}}$ are zero, and either $\lambda_{\{1\}}=1$ or $\lambda_{\{2\}}=1$ or $\lambda_{\{1,2\}}=1 / 2$ according to whether the first, the second, or the third mutual information term is the smallest. Recalling (35), the proof is completed.

\section{EXAMPLES}

Example 1: Consider the DMC $W: \mathcal{X}_{1} \times \mathcal{X}_{2} \rightarrow \mathcal{X}_{3}$ with $\mathcal{X}_{1}=\mathcal{X}_{2}=\mathcal{X}_{3}=\{0,1\}$ and

$$
W\left(x_{3} \mid x_{1}, x_{2}\right)=\mathbb{1}\left(x_{3}=x_{1}+x_{2} \bmod 2\right)
$$

where $\mathbb{1}(\cdot)$ denotes the indicator function. First, note that its capacity region is

$$
\mathcal{C}=\left\{\left(R_{1}, R_{2}\right): 0 \leq R_{1}+R_{2} \leq 1\right\} .
$$

Consider SK generation for $A=\mathcal{M}=\{1,2,3\}$. The lower bound for $C_{S}(\{1,2,3\})$ provided by Theorem 2 is equal to 0.5 since $(R, R)$ belongs to the capacity region $\mathcal{C}$ of the MAC $W$ iff $R \leq 0.5$. This $\mathrm{SK}$ rate is also achieved by simple source emulation; see the Comment following Proposition 5.

The achievability of an SK rate of 0.5 can be seen also separately by the following explicit scheme which generates 1 bit of perfect SK (i.e., $(\epsilon, \delta)$-SK with $\epsilon=\delta=0)$ for $A$ by means of independent transmissions over the DMC by the input terminals using $n=2$ symbols followed by public communication only by the output terminal. Terminal 1 transmits $X_{11}=0$ or 1 w.p. $(0.5,0.5)$ and $X_{12}=0$, while terminal 2 transmits $X_{21}=0$ and
$X_{22}=0$ or 1 w.p. $(0.5,0.5)$, independently of $\left(X_{11}, X_{12}\right)$. Terminal 3 then sends the public message $f_{3}=f_{3}\left(X_{31}, X_{32}\right)=$ $X_{31}+X_{32} \bmod 2$. Clearly, all the terminals can perfectly recover $K=X_{11}$, say, from any $X_{i}^{2}$ and the communication $\mathbf{F}=f_{3}$ while satisfying the secrecy condition

$$
\begin{aligned}
s(K ; \mathbf{F}) & =1-H\left(X_{11} \mid X_{31}+X_{32}\right) \\
& =1-H\left(X_{11} \mid X_{11}+X_{22}\right) \\
& =1-H\left(X_{11}\right)=0 .
\end{aligned}
$$

Thus, $K=X_{11}$ is a perfect SK for $A=\{1,2,3\}$ of rate 0.5 .

Next, for the upper bound, apply Theorem 6 with $D=\emptyset$. Then, the choice $\lambda_{\{1\}}=\lambda_{\{2\}}=\lambda_{\{3\}}=0, \lambda_{\{12\}}=\lambda_{\{13\}}=$ $\lambda_{\{23\}}=0.5$ yields the sum in (26) as $0.5 H\left(X_{3} \mid V\right)$. It follows that $C_{S}(\{1,2,3\}) \leq 0.5$. Thus, $C_{S}(\{1,2,3\})=0.5$.

Furthermore, by Theorem 2, the $\mathrm{PK}$ capacity region $C_{P}(\{1,3\},\{2,3\})$ contains $\mathcal{C}$. In fact, $C_{P}(\{1,3\},\{2,3\})=\mathcal{C}$, which can be seen as follows. Suppose that $C_{P}(\{1,3\},\{2,3\})$ contains a rate pair outside $\mathcal{C}$. By the convexity of the PK region, it contains a rate pair $(R, R)$ with $R>0.5$. Then, again by Theorem $2, R>0.5$ would be an achievable SK rate for $A=\{1,2,3\}$, which contradicts $C_{S}(\{1,2,3\})=0.5$.

Example 2: Consider the DMC $W: \mathcal{X}_{1} \times \mathcal{X}_{2} \rightarrow \mathcal{X}_{3}$ with $\mathcal{X}_{1}=\mathcal{X}_{2}=\{0,1\}, \mathcal{X}_{3}=\{0,1,2\}$ and

$$
W\left(x_{3} \mid x_{1}, x_{2}\right)=\mathbb{1}\left(x_{3}=x_{1}+x_{2}\right) .
$$

Its capacity region is $\left\{\left(R_{1}, R_{2}\right): 0 \leq R_{1}, R_{2} \leq 1, R_{1}+R_{2} \leq\right.$ $1.5\}$. The Corollary of Theorem 2 yields that $C_{S}(\{1,2,3\}) \geq$ 0.75. By the Comment following Proposition 5, the SK rate of 0.75 is achievable also by simple source emulation. On the other hand, Theorem 6 gives the upper bound $C_{S}(\{1,2,3\}) \leq$ $0.5 \log 3=0.7925$. Theorem 7 gives a better upper bound of 0.7911 [23]. The exact value of the SK capacity is unknown.

Next, consider PK generation for $A=\{1,2\}$ with privacy from $D=\{3\}$. Noting in (16) that the only permissible choice of $\lambda \in \Lambda(\{1,2\} \mid\{3\})$ is $\lambda_{\{1\}}=\lambda_{\{2\}}=1$, we get that the righthand side of (16), with $X_{1}, X_{2}$ conditionally independent of $V$, equals $H\left(X_{1} \mid V\right)+H\left(X_{2} \mid V\right)-H\left(X_{3} \mid V\right)$. Thus, by Theorem 4 , it follows that

$$
\begin{aligned}
& C_{P}(\{1,2\} \mid\{3\}) \\
& \geq \max _{P_{V X_{1} X_{2}}=P_{V} P_{X_{1} \mid V} P_{X_{2} \mid V}} H\left(X_{1} \mid V\right)+H\left(X_{2} \mid V\right)-H\left(X_{3} \mid V\right) \\
& =\max _{P_{X_{1} X_{2}}=P_{X_{1}} P_{X_{2}}} H\left(X_{1}\right)+H\left(X_{2}\right)-H\left(X_{3}\right) \\
& =0.5,
\end{aligned}
$$

with the previous maximum attained by $P_{X_{1}}(1)=P_{X_{2}}(1)=$ 0.5 . Thus, the largest $\mathrm{PK}$ rate achievable by general source emulation is 0.5 . The exact value of the PK capacity remains unknown, for Theorem 6 yields only the trivial upper bound 1 .

Example 3: Consider the DMC $W: \mathcal{X}_{1} \times \mathcal{X}_{2} \rightarrow \mathcal{X}_{3}$ with

$$
\begin{gathered}
W\left(0 \mid x_{1}, x_{2}\right)=W\left(1 \mid x_{1}, x_{2}\right)=0.5, \quad \text { if } x_{1}=x_{2}=1 \\
W\left(x_{3} \mid x_{1}, x_{2}\right)=\mathbb{1}\left(x_{3}=x_{1}+x_{2}\right) \quad \text { otherwise. }
\end{gathered}
$$


Its capacity region is the same as that of the MAC in Example 1 . The Corollary of Theorem 2 yields the lower bound $C_{S}(\{1,2,3\}) \geq 0.5$. The same scheme for SK generation for $A=\{1,2,3\}$ as in Example 1 attains an SK rate of 0.5. By Proposition 5, the SK rate of 0.5 is achievable also by general source emulation. However, by the Comment following Proposition 5, it is not achievable by simple source emulation.

By the Corollary of Theorem 4, we obtain as in Example 1 that $C_{S}(\{1,2,3\}) \leq 0.5$, so that $C_{S}(\{1,2,3\})=0.5$.

Example 4: Consider the DMC $W: \mathcal{X}_{1} \times \cdots \times \mathcal{X}_{k} \rightarrow$ $\mathcal{X}_{k+1} \times \cdots \times \mathcal{X}_{m}$ with $\mathcal{X}_{1}=\cdots=\mathcal{X}_{m}=\{0,1\}$ and $W\left(x_{k+1}, \ldots, x_{m} \mid x_{1}, \ldots, x_{k}\right)$

$$
=2^{-(m-k-1)} \mathbb{1}\left(x_{m}=\sum_{i=1}^{m-1} x_{i} \bmod 2\right)
$$

i.e., the DMC outputs at terminals $k+1, \ldots, m-1$ are mutually independent rvs, each distributed uniformly on $\{0,1\}$ regardless of the inputs, and the output at terminal $m$ is the modulo 2 sum of the inputs and the remaining outputs. For SK generation for any $A \subset \mathcal{M}$ with $|A| \geq 2$, the lower bound provided by Theorem 4 yields $C_{S}(A) \geq \frac{1}{|A|-1}$; this SK rate of $\frac{1}{|A|-1}$ is achieved by simple source emulation. Specifically, with the input terminals in $[1, k]$ transmitting i.i.d. repetitions of a $k$-tuple of mutually independent rvs $X_{1}, \ldots, X_{k}$, each distributed uniformly on $\{0,1\}$, the DMC $W$ generates i.i.d. repetitions of an $m$-tuple of rvs $X_{1}, \ldots, X_{m}$, where $X_{1}, \ldots, X_{m-1}$ are mutually independent with each distributed uniformly on $\{0,1\}$ and $X_{m}$ is the modulo 2 sum of $X_{1}, \ldots, X_{m-1}$. In this emulated source model, the largest achievable SK rate for $A$ equals $\frac{1}{A \mid-1}$; see [9, Example 1]. Furthermore, in the explicit scheme provided therein, the key generated for $A$ satisfies the secrecy condition (2) for $D=A^{c}$ with $\delta=0$, thereby constituting a perfect PK for $A$ with privacy from $D=A^{c}$ in addition to being a perfect SK for $A$. Thus, we have $C_{P}\left(A \mid A^{c}\right) \geq \frac{1}{|A|-1}$, too.

For the special case $A=\mathcal{M}$, the achievability of an SK rate of $\frac{1}{m-1}$ can be seen also separately by the following explicit scheme which generates 1 bit of perfect SK for $\mathcal{M}$ by means of independent transmissions over the DMC by the input terminals using $n=m-1$ symbols followed by public communication only by the output terminals but not by the input terminals. Specifically, the input terminal $i \in[1, k]$ transmits over the DMC a sequence $X_{i 1}, \ldots, X_{i n}$ with $X_{i i}$ being $\{0,1\}$-valued w.p. $(0.5,0.5)$ and $X_{i j}=0, j \neq i$; all such sequences are mutually independent. The output terminal $i \in[k+1, m-$ 1] sends a public message $f_{i}=f_{i}\left(X_{i}^{n}\right)$ which is the block $X_{i}^{n}=\left(X_{i 1}, \ldots, X_{i n}\right)$ excluding $X_{i i}$, while the output terminal $m$ sends the public message

$$
f_{m}\left(X_{m}^{n}\right)=\left(X_{m 1}+X_{m 2}, \ldots, X_{m 1}+X_{m n}\right)
$$

where the additions are modulo 2 . It is easily seen that $K=$ $X_{11}$, say, is perfectly recoverable from $X_{i}^{n}$ and the public communication $\mathbf{F}=\left(f_{k+1}, \ldots, f_{m}\right)$. Furthermore, $K$ satisfies the secrecy condition (1) with $\delta=0$, and so constitutes a perfect SK of rate $\frac{1}{m-1}$.
Next, in the upper bound for $C_{S}(A)$ in Theorem 6, we have from (24) that for any $\lambda \in \Lambda(A)$

$$
\begin{aligned}
& G_{A}\left(X_{\mathcal{M}}, V, \lambda\right)-G_{A}\left(X_{[1, k]}, V, \lambda\right) \\
& =\left[H\left(X_{\mathcal{M}} \mid V\right)-\sum_{B \in \mathcal{B}(A)} \lambda_{B} H\left(X_{B} \mid X_{B^{c}}, V\right)\right] \\
& -\left[H\left(X_{[1, k]} \mid V\right)-\sum_{B \in \mathcal{B}(A)} \lambda_{B} H\left(X_{[1, k] \cap B} \mid X_{[1, k] \cap B^{c}}, V\right)\right] \\
& =\left[H\left(X_{\mathcal{M}} \mid V\right)-\sum_{B \in \mathcal{B}(A)} \lambda_{B}\left(H\left(X_{\mathcal{M}} \mid V\right)-H\left(X_{B^{c}} \mid V\right)\right)\right] \\
& -\left[H\left(X_{[1, k]} \mid V\right)-\sum_{B \in \mathcal{B}(A)} \lambda_{B}\left(H\left(X_{[1, k]} \mid V\right)\right.\right. \\
& \left.\left.-H\left(X_{[1, k] \cap B^{c}} \mid V\right)\right)\right] \\
& =\left(1-\sum_{B \in \mathcal{B}(A)} \lambda_{B}\right)\left(H\left(X_{\mathcal{M}} \mid V\right)-H\left(X_{[1, k]} \mid V\right)\right) \\
& +\sum_{B \in \mathcal{B}(A)} \lambda_{B}\left(H\left(X_{B^{c}} \mid V\right)-H\left(X_{[1, k] \cap B^{c}} \mid V\right)\right) \\
& =\left(1-\sum_{B \in \mathcal{B}(A)} \lambda_{B}\right) H\left(X_{[k+1, m]} \mid X_{[1, k]}\right) \\
& +\sum_{B \in \mathcal{B}(A)} \lambda_{B} H\left(X_{B^{c} \backslash[1, k]} \mid X_{[1, k] \cap B^{c}}, V\right) .
\end{aligned}
$$

Fix $i_{0} \in A$, and consider the choice $\lambda_{B}=\frac{1}{|A|-1}$ for $B=A \backslash\left\{i_{0}\right\}$ or $B=\mathcal{M} \backslash\{i\}$ for some $i \in A \backslash\left\{i_{0}\right\}$, and $\lambda_{B}=0$ else. For this choice of $\lambda \in \Lambda(A)$, and noting that $H\left(X_{[k+1, m]} \mid X_{[1, k]}\right)=m-k-1,(36)$ gives

$$
\begin{aligned}
& G_{A}\left(X_{\mathcal{M}}, V, \lambda\right)-G_{A}\left(X_{[1, k]}, V, \lambda\right) \\
& =\left(1-\frac{|A|}{|A|-1}\right)(m-k-1) \\
& +\frac{1}{|A|-1}\left[H\left(X_{\left(A^{c} \cup\left\{i_{0}\right\}\right) \backslash[1, k]} \mid X_{[1, k] \cap\left(A^{c} \cup\left\{i_{0}\right\}\right)}, V\right)\right. \\
& \left.+\sum_{i \in A \backslash\left\{i_{0}\right\}} H\left(X_{\{i\} \backslash[1, k]} \mid X_{[1, k] \cap\{i\}}, V\right)\right] \\
& \leq-\frac{m-k-1}{|A|-1}+\frac{1}{|A|-1}\left[H\left(X_{\left(A^{c} \cup\left\{i_{0}\right\}\right) \backslash[1, k]}\right)\right. \\
& \left.+\sum_{i \in A \backslash\left\{i_{0}\right\}} H\left(X_{\{i\} \backslash[1, k]}\right)\right] \\
& \leq-\frac{m-k-1}{|A|-1}+\frac{1}{|A|-1} \sum_{i=k+1}^{m} H\left(X_{i}\right) \\
& \leq-\frac{m-k-1}{|A|-1}+\frac{m-k}{|A|-1} \\
& =\frac{1}{|A|-1}
\end{aligned}
$$

noting in the first inequality above that the summand in the last term equals 0 if $i \notin[k+1, m]$.

Thus, $C_{S}(A)=\frac{1}{|A|-1}$, and, in particular, $C_{S}(\mathcal{M})=\frac{1}{m-1}$. Also, $C_{P}\left(A \mid A^{c}\right)=\frac{1}{|A|-1}$, as a PK for $A$ with privacy from any $D \subset A^{c}$ is also an SK for $A$. Observe that Example 1 above is a special case of the present example with $m=3, k=2$, and $A=\mathcal{M}$. 


\section{DISCUSSION}

We have considered secrecy generation for multiaccess channel models whose resources consist of facilities for secure noisy channel transmission from the input to the output terminals, public noiseless communication among all the terminals, and (mutually independent) randomization at the terminals. Our main results are single-letter lower and upper bounds for SK and PK capacities, in Theorems 4 and 6, which agree in special cases but not in general. The general channel model considered here appears more defiant than its special case with a single input for which single-letter characterizations of SK and PK capacities were found in [10].

A familiar technique for approaching channel secrecy problems is by means of source emulation. For instance, the available results on the oblivious transfer capacity of simple channel models are obtained by this technique [2]. Regarding secrecy capacities of channel models, they are known to be achievable by simple source emulation in the case of a single input terminal [10]. For multiple input terminals, the general source emulation introduced in this paper can strictly outperform simple source emulation even for models with a single output terminal. Note that the achievability results proved by means of general source emulation in Theorem 4 use very simple protocols.

We show for a MAC model with a single output, in which all the terminals seek to share secrecy, a necessary and sufficient condition for $R$ to be an achievable SK rate by general source emulation is that $(R, \ldots, R)$ must lie in the capacity region of the MAC; thus, the maximum SK rate achievable by source emulation is the largest such $R$ in the MAC capacity region. A main open question for this special model, as well as for the general channel model, is whether secrecy rates can be achieved beyond those attainable by the simple protocols entailed by general source emulation, by resorting to the complex protocols described in Section II. Even for the special case of Example 2, this question remains unresolved. However, for this case, the general upper bound of Theorem 6 is bettered by that of Theorem 7.

\section{APPENDIX A}

In order to complete the proof of the upper bound (25) in Theorem 6, we show in (28) for every $\lambda=\left\{\lambda_{B}: B \in \mathcal{B}(A \mid D)\right\} \in$ $\Lambda(A \mid D)$ and $i \in D^{c}$ that

$$
\begin{aligned}
& H\left(U_{\mathcal{M}}, X_{\mathcal{M}}^{n} \mid U_{D}, X_{D}^{n}, \tilde{\mathbf{F}}^{(n)}\right) \\
& -\sum_{B \in \mathcal{B}(A \mid D)} \lambda_{B} H\left(U_{B}, X_{B}^{n} \mid U_{B^{c}}, X_{B^{c}}^{n}, \tilde{\mathbf{F}}^{(n)}\right) \\
& \leq \sum_{t=1}^{n}\left[\left(H\left(X_{\mathcal{M} t} \mid X_{D t}\right)-\sum_{B \in \mathcal{B}(A \mid D)} \lambda_{B} H\left(X_{B t} \mid X_{B^{c} t}\right)\right)\right. \\
& -\left(H\left(X_{([1, k]) t} \mid X_{D t}\right)\right. \\
& \left.-\sum_{B \in \mathcal{B}(A \mid D)} \lambda_{B} H\left(X_{([1, k] \cap B) t} \mid X_{\left([1, k] \cap B^{c}\right) t}, X_{D t}\right)\right) \\
& \left.+\sum_{B \in \mathcal{B}(A \mid D): B \not \nexists i} \lambda_{B} I\left(X_{D t} \wedge X_{([1, k] \cap D) t} \mid X_{\left([1, k] \cap B^{c}\right) t}\right)\right]
\end{aligned}
$$

and observe that the right side above equals the expression in the claimed bound in (29).

As in [10, Appendix B], the left side of (A1) equals

$$
\begin{aligned}
& \left(1-\lambda_{\text {sum }}\right) H\left(U_{\mathcal{M}}, X_{\mathcal{M}}^{n}\right)-H\left(U_{D}, X_{D}^{n}, \tilde{\mathbf{F}}^{(n)}\right) \\
& +\sum_{B \in \mathcal{B}(A \mid D)} \lambda_{B} H\left(U_{B^{c}}, X_{B^{c}}^{n}, \tilde{\mathbf{F}}^{(n)}\right)
\end{aligned}
$$

where

$$
\lambda_{\text {sum }}=\sum_{B \in \mathcal{B}(A \mid D)} \lambda_{B} \geq 1
$$

Considering the separate terms in (A2), the counterparts of (B4), (B5), and (B7) in [10, Appendix B] are

$$
\begin{aligned}
H\left(U_{\mathcal{M}}, X_{\mathcal{M}}^{n}\right) & =H\left(U_{\mathcal{M}}\right)+\sum_{t=1}^{n} H\left(X_{\mathcal{M} t} \mid U_{\mathcal{M}}, X_{\mathcal{M}}^{t-1}\right) \\
& =H\left(U_{\mathcal{M}}\right)+\sum_{t=1}^{n} H\left(X_{\mathcal{M} t} \mid X_{([1, k]) t}\right)
\end{aligned}
$$

since $X_{([1, k]) t}$ is a function of $\left(U_{[1, k]}, F^{t-1}\right)=$ $\left(U_{[1, k]}, U_{D}, X_{D}^{t-1}, \tilde{F}^{t-1}\right)$ and so is determined by $\left(U_{\mathcal{M}}, X_{\mathcal{M}}^{t-1}\right)$

$$
\begin{aligned}
H\left(U_{D}, X_{D}^{n}, \tilde{\mathbf{F}}^{(n)}\right) & =H\left(U_{D}\right)+\sum_{t=1}^{n} H\left(X_{D t} \mid U_{D}, X_{D}^{t-1}, \tilde{F}^{t-1}\right) \\
& +\sum_{t=1}^{n} H\left(\tilde{F}_{t} \mid U_{D}, X_{D}^{t}, \tilde{F}^{t-1}\right) ;
\end{aligned}
$$

and

$$
\begin{aligned}
H\left(U_{B^{c}}, X_{B^{c}}^{n}, \tilde{\mathbf{F}}^{(n)}\right) & =H\left(U_{B^{c}}\right) \\
& +\sum_{t=1}^{n} H\left(X_{B^{c} t} \mid U_{B^{c}}, X_{B^{c}}^{t-1}, \tilde{F}^{t-1}\right) \\
& +\sum_{t=1}^{n} H\left(\tilde{F}_{t} \mid U_{B^{c}}, X_{B^{c}}^{t}, \tilde{F}^{t-1}\right) .
\end{aligned}
$$

By (A2)-(A6), the left side of (A1) decomposes as $E_{1}+E_{2}+E_{3}$ where

$$
E_{1}=\left(1-\lambda_{\text {sum }}\right) H\left(U_{\mathcal{M}}\right)-H\left(U_{D}\right)+\sum_{B \in \mathcal{B}(A \mid D)} \lambda_{B} H\left(U_{B^{c}}\right)
$$

and

$$
\begin{aligned}
E_{3} & =\sum_{t=1}^{n}\left[-H\left(\tilde{F}_{t} \mid U_{D}, X_{D}^{t}, \tilde{F}^{t-1}\right)\right. \\
& \left.+\sum_{B \in \mathcal{B}\left(A^{\prime} D\right)} \lambda_{B} H\left(\tilde{F}_{t} \mid U_{B^{c}}, X_{B^{c}}^{t}, \tilde{F}^{t-1}\right)\right]
\end{aligned}
$$

are the same as in [10, Appendix B], while

$$
\begin{aligned}
E_{2} & =\sum_{t=1}^{n}\left[\left(1-\lambda_{\text {sum }}\right) H\left(X_{\mathcal{M} t} \mid X_{([1, k]) t}\right)\right. \\
& -H\left(X_{D t} \mid U_{D}, X_{D}^{t-1}, \tilde{F}^{t-1}\right) \\
& \left.+\sum_{B \in \mathcal{B}(A \mid D)} \lambda_{B} H\left(X_{B^{c} t} \mid U_{B^{c}}, X_{B^{c}}^{t-1}, \tilde{F}^{t-1}\right)\right] .
\end{aligned}
$$


By [10, Appendix B], $E_{1}=0$ and $E_{3} \leq 0$. Turning to $E_{2}$, we claim that for each $1 \leq t \leq n$, the $t$ th term of the sum in $E_{2}$, denoted by $E_{2 t}$, satisfies

$$
\begin{aligned}
E_{2 t} & \leq\left[\left(H\left(X_{\mathcal{M} t} \mid X_{D t}\right)-\sum_{B \in \mathcal{B}(A \mid D)} \lambda_{B} H\left(X_{B t} \mid X_{B^{c} t}\right)\right)\right. \\
& -\left(H\left(X_{([1, k]) t} \mid X_{D t}\right)\right. \\
& \left.-\sum_{B \in \mathcal{B}(A \mid D)} \lambda_{B} H\left(X_{([1, k] \cap B) t} \mid X_{\left.([1, k]) \cap B^{c}\right) t}, X_{D t}\right)\right) \\
& \left.+\sum_{B \in \mathcal{B}(A \mid D): B \not \supset i} \lambda_{B} I\left(X_{D t} \wedge X_{([1, k] \cap B) t} \mid X_{\left([1, k] \cap B^{c}\right) t}\right)\right]
\end{aligned}
$$

proving which will establish (A1).

For every $i \in D^{c}$, the first term of $E_{2 t}$ in (A7) is

$$
\begin{aligned}
\left(1-\lambda_{\text {sum }}\right) & H\left(X_{\mathcal{M} t} \mid X_{([1, k]) t}\right) \\
= & -\sum_{B \in \mathcal{B}(A \mid D): B \not i} \lambda_{B} H\left(X_{\mathcal{M} t} \mid X_{([1, k]) t}\right) \text { (A9) }
\end{aligned}
$$

while the third term is

$$
\begin{aligned}
& \sum_{B \in \mathcal{B}(A \mid D)} \lambda_{B} H\left(X_{B^{c} t} \mid U_{B^{c}}, X_{B^{c}}^{t-1}, \tilde{F}^{t-1}\right) \\
\leq & \sum_{B \in \mathcal{B}(A \mid D): B \not \supset i} \lambda_{B} H\left(X_{\left(B^{c} \backslash[1, k]\right) t} \mid X_{\left([1, k] \cap B^{c}\right) t}\right) \\
+ & \sum_{B \in \mathcal{B}(A D): B \ni i} \lambda_{B} H\left(X_{B^{c} t} \mid U_{B^{c}}, X_{B^{c}}^{t-1}, \tilde{F}^{t-1}\right)
\end{aligned}
$$

where the inequality holds since $X_{\left([1, k] \cap B^{c}\right) t}$ is a function of $\left(U_{[1, k] \cap B^{c}}, F^{t-1}\right)=\left(U_{[1, k] \cap B^{c}}, U_{D}, X_{D}^{t-1}, \tilde{F}^{t-1}\right)$ and so is determined by $\left(U_{B^{c}}, X_{B^{c}}^{t-1}, \tilde{F}^{t-1}\right)$. Furthermore, since $B \in$ $\mathcal{B}(A \mid D)$ implies $B^{c} \supset D$

$$
\begin{aligned}
H\left(X_{B^{c} t} \mid U_{B^{c}},\right. & \left.X_{B^{c}}^{t-1}, \tilde{F}^{t-1}\right)=H\left(X_{D t} \mid U_{B^{c}}, X_{B^{c}}^{t-1}, \tilde{F}^{t-1}\right) \\
& +H\left(X_{B^{c} t} \mid X_{D t}, U_{B^{c}}, X_{B^{c}}^{t-1}, \tilde{F}^{t-1}\right) \\
& \leq H\left(X_{D t} \mid U_{D}, X_{D}^{t-1}, \tilde{F}^{t-1}\right)+H\left(X_{B^{c} t} \mid X_{D t}\right) \\
& -I\left(X_{B^{c} t} \wedge U_{B^{c}}, X_{B^{c}}^{t-1}, \tilde{F}^{t-1} \mid X_{D t}\right)
\end{aligned}
$$

so that in the right side of (A10)

$$
\begin{aligned}
& \sum_{B \in \mathcal{B}\left(A^{\prime} D\right): B \ni i} \lambda_{B} H\left(X_{B^{c} t} \mid U_{B^{c}}, X_{B^{c}}^{t-1}, \tilde{F}^{t-1}\right) \\
\leq & \sum_{B \in \mathcal{B}(A \mid D): B \ni i} \lambda_{B}\left[H\left(X_{D t} \mid U_{D}, X_{D}^{t-1}, \tilde{F}^{t-1}\right)\right. \\
+ & \left.H\left(X_{B^{c} t} \mid X_{D t}\right)-I\left(X_{B^{c} t} \wedge U_{B^{c}}, X_{B^{c}}^{t-1}, \tilde{F}^{t-1} \mid X_{D t}\right)\right] .
\end{aligned}
$$

Combining (A9), (A10), and (A11), we get that $E_{2 t}$ is bounded above as

$$
\begin{aligned}
E_{2 t} & \leq-\sum_{B \in \mathcal{B}(A \mid D): B \not \supset i} \lambda_{B}\left[H\left(X_{\mathcal{M} t} \mid X_{([1, k]) t}\right)\right. \\
& \left.-H\left(X_{\left(B^{c} \backslash[1, k]\right) t} \mid X_{\left([1, k] \cap B^{c}\right) t}\right)\right] \\
& +\sum_{B \in \mathcal{B}(A \mid D): B \ni i} \lambda_{B}\left[H\left(X_{B^{c} t} \mid X_{D t}\right)\right. \\
& \left.-I\left(X_{B^{c} t} \wedge U_{B^{c}}, X_{B^{c}}^{t-1}, \tilde{F}^{t-1} \mid X_{D t}\right)\right]
\end{aligned}
$$

Now, we observe in the right side of (A12) that the summands in the first and second sums, respectively, are

$$
\begin{aligned}
& H\left(X_{\mathcal{M} t}\right)-H\left(X_{[1, k] t}\right)-H\left(X_{B^{c} t}\right)+H\left(X_{\left([1, k] \cap B^{c}\right) t}\right) \\
& =H\left(X_{B t} \mid X_{B^{c} t}\right)-H\left(X_{([1, k] \cap B) t} \mid X_{\left([1, k] \cap B^{c}\right) t}\right) \\
& =H\left(X_{B t} \mid X_{B^{c} t}\right)-H\left(X_{([1, k] \cap B) t} \mid X_{\left([1, k] \cap B^{c}\right) t}, X_{D t}\right) \\
& -I\left(X_{D t} \wedge X_{([1, k] \cap B) t} \mid X_{\left([1, k] \cap B^{c}\right) t}\right)
\end{aligned}
$$

and

$$
\begin{aligned}
& H\left(X_{B^{c} t}\right)-H\left(X_{D t}\right) \\
& -I\left(X_{B^{c} t} \wedge X_{\left([1, k] \cap B^{c}\right) t}, U_{B^{c}}, X_{B^{c}}^{t-1}, \tilde{F}^{t-1} \mid X_{D t}\right) \\
& \leq H\left(X_{B^{c} t}\right)-H\left(X_{D t}\right)-I\left(X_{B^{c} t} \wedge X_{\left.(1, k] \cap B^{c}\right) t} \mid X_{D t}\right) \\
& =H\left(X_{B^{c} t}\right)-H\left(X_{D t}\right)-H\left(X_{\left([1, k] \cap B^{c}\right) t} \mid X_{D t}\right) \\
& =H\left(X_{\mathcal{M} t}\right)-H\left(X_{B t} \mid X_{B^{c} t}\right)-H\left(X_{D t}\right)-H\left(X_{([1, k]) t} \mid X_{D t}\right) \\
& +H\left(X_{([1, k] \cap B) t} \mid X_{\left([1, k] \cap B^{c}\right) t}, X_{D t}\right)
\end{aligned}
$$

where the insertion of $X_{\left([1, k] \cap B^{c}\right) t}$ in the first expression in (A14) is permissible for the reason in the passage following (A10).

Finally, from (A12), (A13), and (A14), we get that for every $i \in D^{c}$

$$
\begin{aligned}
& E_{2 t} \leq-\sum_{B \in \mathcal{B}(A \mid D): B \not \supset i} \lambda_{B}\left[H\left(X_{B t} \mid X_{B^{c} t}\right)\right. \\
&-H\left(X_{([1, k] \cap B) t} \mid X_{\left.([1, k]) \cap B^{c}\right) t}, X_{D t}\right) \\
&\left.-I\left(X_{D t} \wedge X_{([1, k] \cap B) t} \mid X_{\left([1, k] \cap B^{c}\right) t}\right)\right] \\
&+\sum_{B \in \mathcal{B}(A \mid D): B \ni i} \lambda_{B}\left[H\left(X_{\mathcal{M} t}\right)-H\left(X_{B t} \mid X_{B^{c} t}\right)-H\left(X_{D t}\right)\right. \\
&-\left.H\left(X_{([1, k]) t} \mid X_{D t}\right)+H\left(X_{([1, k] \cap B) t} \mid X_{\left.([1, k]) \cap B^{c}\right) t}, X_{D t}\right)\right] \\
&= {\left[\left(H\left(X_{\mathcal{M} t} \mid X_{D t}\right)-\sum_{B \in \mathcal{B}(A D)} \lambda_{B} H\left(X_{B t} \mid X_{B^{c} t}\right)\right)\right.} \\
&-\left(H\left(X_{([1, k]) t} \mid X_{D t}\right)\right. \\
&-\left.\sum_{B \in \mathcal{B}(A \mid D)} \lambda_{B} H\left(X_{([1, k] \cap B) t} \mid X_{\left.([1, k]) \cap B^{c}\right) t}, X_{D t}\right)\right) \\
&+\left.\sum_{B \in \mathcal{B}(A \mid D): B \not \nexists i} \lambda_{B} I\left(X_{D t} \wedge X_{([1, k] \cap B) t} \mid X_{\left([1, k] \cap B^{c}\right) t}\right)\right]
\end{aligned}
$$

which is (A8). 
For the proof of Theorem 7, a refinement of the bounding of $E_{2}$ in (A7) is needed for the special case of a MAC in class $\mathcal{W}$ with $A=\mathcal{M}=\{1,2,3\}, D=\emptyset, \tilde{F}^{t}=F^{t}$.

In this case, the $t$ th term of the sum in (A7) is

$$
\begin{aligned}
E_{2 t} & =\left(1-\lambda_{\text {sum }}\right) H\left(X_{3 t} \mid X_{1 t}, X_{2 t}\right) \\
& +\sum_{B \in \mathcal{B}(\mathcal{M})} \lambda_{B} H\left(X_{B^{c} t} \mid U_{B^{c}}, X_{B^{c}}^{t-1}, F^{t-1}\right) .
\end{aligned}
$$

Here, $H\left(X_{B^{c} t} \mid U_{B^{c}}, X_{B^{c}}^{t-1}, F^{t-1}\right)=0$ if $B \ni 3$ because $X_{i t}$ is a function of $U_{i}, F^{t-1}$ if $i \in B^{c} \subset\{1,2\}$. For the same reason, if $B=\{2\}$, then

$$
\begin{aligned}
& H\left(X_{B^{c} t} \mid U_{B^{c}}, X_{B^{c}}^{t-1}, F^{t-1}\right) \\
& =H\left(X_{3 t} \mid X_{1 t}, U_{1}, U_{3},\left(X_{1}, X_{3}\right)^{t-1}, F^{t-1}\right) \\
& \leq H\left(X_{3 t} \mid X_{1 t}, U_{3},\left(X_{1}, X_{3}\right)^{t-1}, F^{t-1}\right) .
\end{aligned}
$$

If $B=\{1\}$, a similar bound holds with $X_{1}$ and $X_{2}$ interchanged, and the assumption $X_{1}=\phi\left(X_{2}, X_{3}\right)$ allows further bounding by $H\left(X_{3 t} \mid X_{2 t}, U_{3},\left(X_{1}, X_{3}\right)^{t-1}, F^{t-1}\right)$. Using this, and that (see (A9))

$$
\left(1-\lambda_{\text {sum }}\right) H\left(X_{3 t} \mid X_{1 t}, X_{2 t}\right)=-\sum_{B \not 3} \lambda_{B} H\left(X_{3 t} \mid X_{1 t}, X_{2 t}\right)
$$

where the conditional entropy is unaltered by further conditioning on the past, we obtain

$$
\begin{aligned}
E_{2 t} & \leq \lambda_{\{1\}} I\left(X_{1 t} \wedge X_{3 t} \mid X_{2 t},\left(X_{1}, X_{3}\right)^{t-1}, F^{t-1}, U_{3}\right) \\
& +\lambda_{\{2\}} I\left(X_{2 t} \wedge X_{3 t} \mid X_{1 t},\left(X_{1}, X_{3}\right)^{t-1}, F^{t-1}, U_{3}\right) \\
& +\lambda_{\{1,2\}} I\left(X_{1 t}, X_{2 t} \wedge X_{3 t} \mid X_{3}^{t-1}, F^{t-1}, U_{3}\right) .
\end{aligned}
$$

\section{APPENDIX B}

The proof of the nonnegativity of (23) relies on the following technical lemma; that of (22) follows with $D=\emptyset$.

Lemma $B$ : Let $L=\left\{i_{1}, \ldots, i_{l}\right\} \subset \mathcal{M}, i_{1}<\cdots<i_{l}$, and $D \subset \mathcal{M}$ be arbitrary sets with $L \backslash D \neq \emptyset$. For rvs $X_{\mathcal{M}}, Y$, and for every collection $\lambda=\left\{\lambda_{B}: B \subset D^{c}\right\}$ of weights $0 \leq \lambda_{B} \leq 1$ satisfying

$$
\sum_{B \subset D^{c}: B \ni i} \lambda_{B}=1 \text { for all } i \in L \backslash D
$$

it holds that

$$
\sum_{B \subset D^{c}} \lambda_{B} H\left(X_{L \cap B} \mid X_{L \cap B^{c}}, Y\right) \leq H\left(X_{L \backslash D} \mid Y\right)
$$

Comment: Lemma B is a special case of Lemma B1 in [10] and also of Theorem 1 in [16].

Proof: We have

$$
\begin{aligned}
& \sum_{B \subset D^{c}} \lambda_{B} H\left(X_{L \cap B} \mid X_{L \cap B^{c}}, Y\right) \\
= & \sum_{B \subset D^{c}} \lambda_{B} \sum_{j: i_{j} \in L \cap B} H\left(X_{i_{j}} \mid X_{\left\{i_{1}, \ldots, i_{j-1}\right\} \cap B}, X_{L \cap B^{c}}, Y\right) \\
\leq & \sum_{B \subset D^{c}} \sum_{j: i_{j} \in L \cap B} \lambda_{B} H\left(X_{i_{j}} \mid X_{\left\{i_{1}, \ldots, i_{j-1}\right\}}, Y\right) \\
= & \sum_{j: i_{j} \in L \backslash D} \sum_{B \subset D^{c}: B \ni i_{j}} \lambda_{B} H\left(X_{i_{j}} \mid X_{\left\{i_{1}, \ldots, i_{j-1}\right\}}, Y\right) \\
= & \sum_{j: i_{j} \in L \backslash D} H\left(X_{i_{j}} \mid X_{\left\{i_{1}, \ldots, i_{j-1}\right\}}, Y\right), \text { by }(B 1) \\
\leq & \sum_{j: i_{j} \in L \backslash D} H\left(X_{i_{j}} \mid X_{\left\{i_{1}, \ldots, i_{j-1}\right\} \backslash D}, Y\right) \\
= & H\left(X_{L \backslash D} \mid Y\right) .
\end{aligned}
$$

The claimed nonnegativity of (23) follows upon taking $L=$ $X_{[1, k]}$ and $Y=\left(X_{D}, V\right)$ in Lemma B. This lemma also provides a formal proof of the nonnegativity of (8) and (9), with $L=X_{\mathcal{M}}$

\section{APPENDIX C}

Lemma $C$ : For a MAC $W$ with two inputs and one output, and any protocol ${ }^{3}$ as in Section II, it holds for $t \in\{1, \ldots, n\}$ that

$$
\begin{aligned}
& I\left(U_{1} \wedge U_{2} \mid\left(X_{1}, X_{3}\right)^{t}, F^{t}, U_{3}\right) \\
& -I\left(U_{1} \wedge U_{2} \mid\left(X_{1}, X_{3}\right)^{t-1}, F^{t-1}, U_{3}\right) \\
& \leq-I\left(X_{1 t} \wedge X_{2 t} \mid\left(X_{1}, X_{3}\right)^{t-1}, F^{t-1}, U_{3}\right) .
\end{aligned}
$$

Proof: First we show that

$$
\begin{aligned}
& I\left(U_{1} \wedge U_{2} \mid\left(X_{1}, X_{3}\right)^{t}, F^{t}, U_{3}\right) \\
& \leq I\left(U_{1} \wedge U_{2} \mid\left(X_{1}, X_{3}\right)^{t}, F^{t-1}, U_{3}\right) .
\end{aligned}
$$

Recall that the communication $F_{t}$ in interval $t$ equals a sequence $f_{1}, \ldots, f_{3 r}$ of messages sent consecutively by the terminals $1,2,3$ in $r$ rounds. If message $f_{j}$ is sent by terminal 1 , then

$$
\begin{aligned}
& I\left(U_{1} \wedge U_{2} \mid\left(X_{1}, X_{3}\right)^{t}, F^{t-1}, f^{j}, U_{3}\right) \\
& \leq I\left(U_{1}, f_{j} \wedge U_{2} \mid\left(X_{1}, X_{3}\right)^{t}, F^{t-1}, f^{j-1}, U_{3}\right) \\
& =I\left(U_{1} \wedge U_{2} \mid\left(X_{1}, X_{3}\right)^{t}, F^{t-1}, f^{j-1}, U_{3}\right)
\end{aligned}
$$

\footnotetext{
${ }^{3}$ The independence of $U_{1}, U_{2}, U_{3}$ is not needed for this lemma.
} 
since $f_{j}$ is a function of $U_{1}$ and the prior communication $F^{t-1}, f^{j-1}$. The inequality

$$
\begin{aligned}
& I\left(U_{1} \wedge U_{2} \mid\left(X_{1}, X_{3}\right)^{t}, F^{t-1}, f^{j}, U_{3}\right) \\
& \leq I\left(U_{1} \wedge U_{2} \mid\left(X_{1}, X_{3}\right)^{t}, F^{t-1}, f^{j-1}, U_{3}\right)
\end{aligned}
$$

follows similarly when $f_{j}$ is sent by terminal 2 , and holds with equality when $f_{j}$ is sent by terminal 3 for then $f_{j}$ is a function of $U_{3}, X_{3}^{t}, F^{t-1}, f^{j-1}$. The validity of these inequalities for $j=$ $1,2,3$ implies (C2).

The assertion $(\mathrm{C} 1)$ follows from $(\mathrm{C} 2)$ and the inequality

$$
\begin{aligned}
& I\left(U_{1} \wedge U_{2} \mid\left(X_{1}, X_{3}\right)^{t}, F^{t-1}, U_{3}\right) \\
& -I\left(U_{1} \wedge U_{2} \mid\left(X_{1}, X_{3}\right)^{t-1}, F^{t-1}, U_{3}\right) \\
& \leq-I\left(X_{1 t} \wedge X_{2 t} \mid\left(X_{1}, X_{3}\right)^{t-1}, F^{t-1}, U_{3}\right) .
\end{aligned}
$$

The latter is a version of the dependence balance bound [14, eq. (1)]; note that the conditioning on $F^{t-1}, U_{3}$ here does not affect the proof.

\section{REFERENCES}

[1] R. Ahlswede and I. Csiszár, "Common randomness in information theory and cryptography - I: Secret sharing," IEEE Trans. Inf. Theory, vol. 39, no. 4, pp. 1121-1132, Jul. 1993.

[2] R. Ahlswede and I. Csiszár, "On oblivious transfer capacity," in Proc. IEEE Int. Symp. Inf. Theory, Nice, France, Jun. 2007, pp. 2061-2064.

[3] C. H. Bennett, G. Brassard, and J. M. Robert, "Privacy amplification by public discussion," SIAM J. Comput., vol. 17, no. 2, pp. 210-229, 1988.

[4] C. H. Bennett, G. Brassard, C. Crépeau, and U. M. Maurer, "Generalized privacy amplification,” IEEE Trans. Inf. Theory, vol. 41, no. 6, pp. 1915-1923, Nov. 1995.

[5] T. M. Cover and C. S. K. Leung, "An achievable rate region for the multiple-access channel with feedback," IEEE Trans. Inf. Theory, vol. 27, no. 3, pp. 292-298, May 1981.

[6] I. Csiszár, "Almost independence and secrecy capacity,” Probl. Pered. Inform. (Special Issue Devoted to M.S. Pinsker), vol. 32, no. 1, pp. 48-57, 1996.

[7] I. Csiszár and J. Körner, "Broadcast channels with confidential messages," IEEE Trans. Inf. Theory, vol. 24, no. 3, pp. 339-348, May 1978.

[8] I. Csiszár and J. Körner, Information Theory: Coding Theorems for Discrete Memoryless Systems, 2nd ed. Cambridge: Cambridge Univ. Press, 2011.

[9] I. Csiszár and P. Narayan, "The secret key capacity of multiple terminals," IEEE Trans. Inf. Theory, vol. 50, no. 12, pp. 3047-3061, Dec. 2004.

[10] I. Csiszár and P. Narayan, "Secrecy capacities for multiterminal channel models," IEEE Trans. Inf. Theory, vol. 54, no. 6, pp. 2437-2452, Jun. 2008.

[11] I. Csiszár and P. Narayan, "Secrecy generation for multiple input multiple output channel models," in Proc. IEEE Int. Symp. Inf. Theory, Seoul, Korea, Jun./Jul. 2009, pp. 2447-2451

[12] A. Gohari and V. Anantharam, "Information-theoretic key agreement of multiple terminals-Part I: Source model," IEEE Trans. Inf. Theory, vol. 56, no. 8, pp. 3973-3996, Aug. 2010.

[13] A. Gohari and V. Anantharam, "Information-theoretic key agreement of multiple terminals-Part II: Channel model," IEEE Trans. Inf. Theory, vol. 56, no. 8, pp. 3997-4010, Aug. 2010.

[14] A. P. Hekstra and F. M. J. Willems, "Dependence balance bounds for multiple access channels with feedback and equal output two-way channels," in Proc. Zesde Symp. Over Informatie Theory de Benelux, Mierlo, The Netherlands, May 1985, pp. 193-198.

[15] M. Madiman and A. Barron, "Generalized entropy power inequalities and monotonicity properties of information," IEEE Trans. Inf. Theory, vol. 53 , no. 7 , pp. $2317-2329$, Jul. 2007.
[16] M. Madiman and P. Tetali, "Information inequalities for joint distributions, with interpretations and applications," IEEE Trans. Inf. Theory, vol. 56, no. 6, pp. 2699-2713, Jun. 2010.

[17] U. M. Maurer, "Secret key agreement by public discussion from common information," IEEE Trans. Inf. Theory, vol. 39, no. 3, pp. 733-742, May 1993.

[18] U. M. Maurer, "The Strong Secret Key Rate of Discrete Random Triples," in Communications and Cryptography: Two Sides of One Tapestry, R. E. Blahut, D. J. Costello Jr., U. Maurer, and T. Mittelholzer, Eds. Norwell, MA: Kluwer, 1994, ch. 26, pp. 271-285.

[19] U. M. Maurer and S. Wolf, "Unconditionally secure key agreement and the intrinsic conditional information," IEEE Trans. Inf. Theory, vol. 45, no. 2, pp. 499-514, Mar. 1999.

[20] U. M. Maurer and S. Wolf, "Information-theoretic key agreement: From weak to strong secrecy for free," in Proc. EUROCRYPT, 2000, pp. $352-368$.

[21] R. Renner and S. Wolf, "New bounds in secret-key agreement: The gap between formation and secrecy extraction," in Proc. EUROCRYPT, 2003, pp. 562-577.

[22] F. M. J. Willems, "The feedback capacity region of a class of discrete memoryless multiple access channels," IEEE Trans. Inf. Theory, vol. 28, no. 1, pp. 93-95, Jan. 1982.

[23] F. M. J. Willems, "On multiple access channels with feedback," IEEE Trans. Inf. Theory, vol. 30, no. 6, pp. 842-845, Nov. 1984.

[24] A. D. Wyner, "The wiretap channel," Bell Syst. Tech. J., vol. 54, pp. 1355-1387, 1975.

Imre Csiszár received the diploma in mathematics from the L. Eötvös University, Budapest, in 1961, and the Doctor of Mathematical Science degree from the Hungarian Academy of Sciences in 1977. He has been with the Mathematical Institute, now called Rényi Institute, of the Hungarian Academy of Sciences since 1961, being Head of the Information Theory Group there from 1968 to 2008. Also, he had been Professor of Mathematics at the L. Eötvös University, Budapest, and the University of Technology and Economics, Budapest; currently, he is Professor Emeritus of the latter. He has held visiting professorships at several universities in Europe and the US. His research interests are centered on information theory and its applications in probability and statistics. He is coauthor of the books Information Theory: Coding Theorems for Discrete Memoryless Systems (New York: Academic Press, 1981; second edition Cambridge: Cambridge University Press, 2012) and Information Theory and Statistics: A Tutorial (Hanover: now Publishers, 2004).

I. Csiszár is Regular Member of the Hungarian Academy of Sciences. He is Honorary President of the J.Bolyai Mathematical Society (Hungarian Mathematical Society), and Fellow of the IEEE. He has been recipient of several academic awards, including the 1988 Prize Paper Award of the IEEE IT Society, the Award for Interdisciplinary Research of the Hungarian Academy of Sciences in 1989, the Shannon Award of the IEEE IT Society in 1996, the Bolzano Medal of the Czech Academy of Sciences in 2006, and the Széchenyi Prize of the Hungarian Republic in 2007.

Prakash Narayan received the Bachelor of Technology degree in Electrical Engineering from the Indian Institute of Technology, Madras in 1976. He received and the M.S. degree in Systems Science and Mathematics in 1978, and the D.Sc. degree in Electrical Engineering, both from Washington University, St. Louis, MO.

$\mathrm{He}$ is Professor of Electrical and Computer Engineering at the University of Maryland, College Park, with a joint appointment at the Institute for Systems Research. He has held visiting appointments at ETH, Zurich; the Technion, Haifa; the Renyi Institute of the Hungarian Academy of Sciences, Budapest; the University of Bielefeld; the Institute of Biomedical Engineering (formerly LADSEB), Padova; and the Indian Institute of Science, Bangalore. His research interests are in multiuser information theory, communication theory, communication networks, cryptography, and information theory and statistics.

Dr. Narayan has served as Associate Editor for Shannon Theory for the IEEE TRANSACTIONS ON INFORMATION THEORY; was Co-Organizer of the IEEE Workshop on Multi-User Information Theory and Systems, VA (1983); Technical Program Chair of the IEEE/IMS Workshop on Information Theory and Statistics, VA (1994); General Co-Chair of the IEEE International Symposium on Information Theory, Washington, D.C. (2001); and Technical Program Co-Chair of the IEEE Information Theory Workshop, Bangalore (2002). He served as a Member of the Board of Governors of the IEEE Information Theory Society from 2007 to 2012 . 